\title{
PRODUCT MATRIX PROCESSES FOR COUPLED MULTI-MATRIX MODELS AND THEIR HARD EDGE SCALING LIMITS
}

\author{
GERNOT AKEMANN AND EUGENE STRAHOV
}

\begin{abstract}
Product matrix processes are multi-level point processes formed by the singular values of random matrix products. In this paper we study such processes where the products of up to $m$ complex random matrices are no longer independent, by introducing a coupling term and potentials for each product. We show that such a process still forms a multi-level determinantal point processes, and give formulae for the relevant correlation functions in terms of the corresponding kernels.

For a special choice of potential, leading to a Gaussian coupling between the $m$ th matrix and the product of all previous $m-1$ matrices, we derive a contour integral representation for the correlation kernels suitable for an asymptotic analysis of large matrix size $n$. Here, the correlations between the first $m-1$ levels equal that of the product of $m-1$ independent matrices, whereas all correlations with the $m$ th level are modified. In the hard edge scaling limit at the origin of the spectra of all products we find three different asymptotic regimes. The first regime corresponding to weak coupling agrees with the multi-level process for the product of $m$ independent complex Gaussian matrices for all levels, including the $m$-th. This process was introduced by one of the authors and can be understood as a multi-level extension of the Meijer $G$-kernel introduced by Kuijlaars and Zhang. In the second asymptotic regime at strong coupling the point process on level $m$ collapses onto level $m-1$, thus leading to the process of $m-1$ independent matrices. Finally, in an intermediate regime where the coupling is proportional to $n^{\frac{1}{2}}$, we obtain a family of parameter dependent kernels, interpolating between the limiting processes in the weak and strong coupling regime. These findings generalise previous results of the authors and their coworkers for $m=2$.
\end{abstract}

\section{Contents}

1. Introduction

2. Exact results for general coupling and finite matrix size

2.1. Coupled multi-matrix models with general potential functions

2.2. Density of product matrix processes and related biorthogonal ensembles

2.3. Exact formulae for the correlation kernels

2.4. Hierarchy of correlation kernels at finite matrix size

3. Hard edge scaling limits of the multi-level determinantal processes 17

4. The interpolating multi-level determinantal process 20

5. An integration formula for coupled matrices 21

6. Proof of Theorem 2.1 25

Key words and phrases. Products of random matrices, multi-matrix models, multi-level determinantal point processes, interpolating ensembles. 
7. Proof of Proposition 2.5 27

8. Measures given by products of determinants, Eynard-Mehta Theorem, and proof of Theorem 2.7

9. Double contour integral representation for the correlation kernel 33

10. Proof of Theorem $3.2 \quad 41$

10.1. The weak coupling regime 41

10.2. The intermediate coupling regime 42

10.3. The strong coupling regime $\quad 42$

11. Proof of Theorem 4.1 47

References 48

\section{INTRODUCTION}

By a random multi-matrix model one usually means a probability measure defined on a space formed by a collection of matrices of the same type. Probably the best known example is that of Hermitian matrices coupled in a chain considered by Eynard and Mehta [18]. This example represents the simplest case of multi-matrix models known from applications in quantum field theory. Another class of examples consists of multimatrix models of positive Hermitian matrices subject to the Cauchy interaction. This class of multi-matrix models was introduced in Bertola, Gekhtman, and Szmigielski [12], and studied further in Bertola, Gekhtman, and Szmigielski [13, 14, and in Bertola and Bothner [10]. For other examples of multi-matrix models, and for an explanation of their relevance to quantum field theory and to statistical mechanics we refer the reader to Eynard, Kimura, and Ribault [17, Section 2.2.], Filev and O'Connor [19], Bertola, Eynard, Harnad [11], and references therein.

The starting point of the present work is the observation that the problem about the distribution of singular values for a product of independent complex Gaussian matrices can be reformulated in terms of a multi-matrix model. This multi-matrix model can be defined by the probability measure

$$
\frac{1}{Z_{n}} e^{-\sum_{l=1}^{m} \operatorname{Tr}\left[G_{l}^{*} G_{l}\right]} \prod_{l=1}^{m} d G_{l} .
$$

over the set of rectangular complex matrices $\left(G_{1}, \ldots, G_{m}\right)$, where $G_{l}$ is of size $\left(n+\nu_{l}\right) \times$ $\left(n+\nu_{l-1}\right), \nu_{0}=0, \nu_{1} \geq 0, \ldots, \nu_{m-1} \geq 0, d G_{l}$ is the corresponding flat complex Lebesgue measure, and $Z_{n}$ is a normalisation constant. Given this probability measure one can ask about the distribution of complex eigenvalues and of squared singular values of the total product matrix $Y_{m}=G_{m} \cdots G_{1}$. It turns out that the eigenvalues of $Y_{m}$ form a determinantal point process on the complex plane which can be understood as a generalisation of the classical Ginibre ensemble. This fact was first proved in Akemann and Burda [2], see Adhikari, Kishore Reddy, Ram Reddy, and Saha [1], Forrester [21, Ipsen [25], Akemann, Ipsen, and Strahov [6], Forrester and Ipsen [22] for different proofs and extensions of this result. Moreover, it was shown in Akemann, Kieburg, and Wei 
[4], and in Akemann, Ipsen, and Kieburg [5] that the joint probability density function of the squared singular values of $Y_{m}$ forms a determinantal point process, representing a special polynomial ensemble, a notion introduced later by Kuijlaars and Stivigny [30]. A contour integral representation for the correlation kernel of this ensemble was derived in Kuijlaars and Zhang [31], which enabled a detailed analysis of different scaling limits, see Kuijlaars and Zhang [31, Liu, Wang, and Zhang 34].

A natural (multi-level) generalisation of the point process formed by the squared singular values of the total product matrix $Y_{m}$ can be constructed as follows. For each $l \in\{1, \ldots, m\}$, denote by $y_{j}^{l}, j=1, \ldots, n$, the squared singular values of the partial product $Y_{l}=G_{l} \cdots G_{1}$, that is the eigenvalues of $Y_{l}^{*} Y_{l}$. The configuration

$$
\left\{\left(l, y_{j}^{l}\right) \mid l=1, \ldots, m ; j=1, \ldots, n\right\}
$$

of all eigenvalues forms a point process on $\{1, \ldots, m\} \times \mathbb{R}_{>0}$. This process was introduced and studied in Strahov [41, calling it the Ginibre product process. It was shown in [41] that it is a multi-level determinantal point process. Furthermore, a contour integral representation for the correlation kernel of this process was derived, and its hard edge scaling limit computed.

Here, we will drop the assumption that the matrices $G_{1}, \ldots, G_{m}$ are independent as in (1.1), but assume instead that these or their products becomes coupled. We can still construct and investigate the multi-level point process formed by configurations (1.2) and refer to it as the product matrix process associated with $G_{1}, \ldots, G_{m}$. It is an interesting general problem to describe statistical properties of such product matrix processes, their relevant correlation functions, and their scaling limits. Of course it is desirable that it still forms a multi-level determinantal point process, with explicit formulae for the correlation kernel and thus the correlation functions. An example for such a setup is the multi-matrix model for Hurwitz numbers [9]. In this paper we introduce and study a different multi-matrix model of statistically dependent random matrices, also satisfying these requirements. We show (see Theorem 2.7) that these product matrix processes generalise the Ginibre product process studied in Strahov [41]. For a particular choice of potentials the first $m-1$ levels remain that of products of $m-1$ independent random matrices, to which the $m$-th level is coupled. For this example we derive contour integral representations for the relevant correlation kernels, see Theorem 2.8 .

In the hard edge scaling limit at large matrix sizes we distinguish three different asymptotic regimes. In the first regime corresponding to weak coupling, the limiting product matrix process is the same as for the product of $m$ independent complex Gaussian matrices, as described in Strahov [41]. This process can be called the $m$-level Meijer $G$-kernel process, a multi-level extension of the Meijer $G$-kernel process introduced by Kuijlaars and Zhang [31]. In the second asymptotic regime at strong coupling the $m$ th level collapses to the point process on the $(m-1)$-th level. In other words, the correlations between the $m$-th level and the levels up to $m-2$ are the same as the respective correlations between the $(m-1)$-th and the levels up to $m-2$. The correlations between level $m$ and $m-1$ are as if they are on the same level, except at colliding points where we find a contact interaction in form of a Dirac delta function. Finally, 
at intermediate coupling we obtain a limiting process of $m$ levels, interpolating between that in the weak coupling regime and that in the strong coupling regime. All three hard edge scaling limits described above are given by Theorem 3.2 that we consider as the main achievement of the present work.

We note that interpolating ensembles are of great interest in Random Matrix Theory. In the literature one can find examples interpolating between Gaussian ensembles of different symmetry classes - one classical example is that of Pandey and Mehta [40] that interpolates between the Gaussian Orthogonal and Gaussian Unitary Ensemble. Determinantal processes whose edge behaviour interpolates between the Poisson process and the Airy process were considered in Moshe, Neuberger, and Shapiro [37], and studied further by Johansson [29]. In the context of products of random matrices, a determinantal process with $m=2$ interpolating between the classical Bessel-kernel process (at $m=1$ ), and the Meijer $G$-kernel process for the product of $m=2$ independent Gaussian matrices was obtained by the authors in [7, 8], and further extended most recently by Liu [33] and a joint work [3]. However, we are not aware of further examples of multilevel interpolating ensembles, with properties described by Theorem 4.1, In particular, the results mentioned above lead to three different scaling limits for the biorthogonal ensemble formed by the squared singular values of the total product matrix $Y_{m}$ : that of the Meijer $G$-kernel process for the product of $m(m-1)$ independent rectangular matrices with complex Gaussian entries at weak (strong) coupling, and a determinantal process interpolating between these correlation kernels, see Corollary 3.3 ,

This paper is organised as follows. In Section 2 we present exact results at finite matrix sizes. In particular, in Section 2 we define a family of multi-matrix models, and introduce the product matrix process associated with this family. We show that the product matrix process under considerations is a multi-level determinantal process, and give formulae for the correlation kernels. In Section 3 we compute the hard edge scaling limits in our example, corresponding to different asymptotic regimes, and in Section 4 we describe the properties of the obtained interpolating multi-level determinantal process. Sections 5,11 contain the proofs our statements.

Acknowledgements. We are very grateful to Marco Bertola, Tomasz Checinski and Mario Kieburg for discussions and useful comments. The anonymous referee is also thanked for several comments and corrections. This work was supported by the DFG through grant AK35/2-1 and CRC 1283 "Taming uncertainty and profiting from randomness and low regularity in analysis, stochastics and their applications" (G.A.). 


\section{ExACT RESUlts FOR GENERAL COUPLing AND FINITE MATRIX SIZE}

2.1. Coupled multi-matrix models with general potential functions. Fix $a>0$, $b>0$, and consider a multi-matrix model defined by the probability distribution

$$
\begin{aligned}
& \frac{1}{Z_{n}} \exp \left[-a \sum_{l=1}^{m} \operatorname{Tr}\left[G_{l}^{*} G_{l}\right]+b \operatorname{Tr}\left[G_{m} \cdots G_{1}+\left(G_{m} \cdots G_{1}\right)^{*}\right]\right] \\
& \times \exp \left[-\sum_{l=1}^{m} \operatorname{Tr}\left[V_{l}\left(\left(G_{l} \cdots G_{1}\right)^{*}\left(G_{l} \cdots G_{1}\right)\right)\right]\right] \prod_{l=1}^{m} d G_{l},
\end{aligned}
$$

normalised by the constant $Z_{n}$. It depends on $m$ rectangular complex matrices $G_{l}$ is of size $\left(n+\nu_{l}\right) \times\left(n+\nu_{l-1}\right)$ for $l=1, \ldots, m$, with $\nu_{0}=0, \nu_{m}=0, \nu_{1} \geq 0, \ldots, \nu_{m-1} \geq 0$, and where $d G_{l}$ is the corresponding flat complex Lebesgue measure. Here, $V_{l}$ (the potentials) are some scalar positive functions which are continuous and grow fast enough at infinity to guarantee the convergence of the corresponding matrix measure (2.1).

Note that the potentials and in particular the parameter $b$ introduce a coupling amongst the matrices: if $b=0$, and $V_{l}=0 \forall l=1, \ldots, m$, then the matrices $G_{1}, \ldots, G_{m}$ become independent Gaussian random matrices.

The multi-level point process associated with the multi-matrix model (2.1) of coupled random matrices can be constructed as that for the Ginibre product process [41] of independent matrices. Namely denote by $\left\{y_{j}^{l}\right\}_{j=1, \ldots, n}$ the set of squared singular values of matrix $Y_{l}=G_{l} \cdots G_{1}$, these are the eigenvalues of $Y_{l}^{*} Y_{l}$. The configuration of all these eigenvalues forms a point process on $\{1, \ldots, m\} \times \mathbb{R}_{>0}$. We will call this point process the product matrix process corresponding to the multi-matrix model (2.1).

Let us indicate some particular cases of the multi-matrix model (2.1) studied previously. At zero coupling $b=0$, and $V_{1}=\ldots=V_{m}=0$, we are dealing with $m$ independent complex Ginibre matrices, and the product matrix process turns into the Ginibre product process studied in [41] (by rescaling all matrices $G_{l}$ we can set $a=1$ ). A second example with $b=0, V_{1}=\ldots=V_{m-1}=0$ and non-vanishing potential $V_{m}$ was considered in [9] in order to study so-called hypergeometric Hurwitz numbers. As a further feature a non-trivial covariance matrix $\Sigma^{-1}$ is introduced there in the measure for the first matrix, replacing $\operatorname{Tr}\left[G_{1}^{*} G_{1}\right]$ by $\operatorname{Tr}\left[\Sigma^{-1} G_{1}^{*} G_{1}\right]$. While in [9] loop equations were applied we will indicate below that this model defines a multi-level point process.

For $m=2$ matrices with non vanishing coupling $b \neq 0$, with both potential functions equal to zero, $V_{1}=V_{2}=0$, and parameters given by $a=\frac{1+\mu}{2 \mu}, b=\frac{1-\mu}{2 \mu}$, we obtain a model of two coupled matrices introduced by Osborn [39] in the context of QCD with a baryon chemical potential $\mu$. For this model, the distribution of squared singular values of the product matrix $Y_{2}$ was studied by the authors in [7, 8]. By its construction in [39], the two coupled matrices can be defined in terms of linear combinations of independent Gaussian matrices, too. Namely, let $A, B$ be two independent matrices of sizes $n \times L$, with i.i.d. standard complex Gaussian entries. Define random matrices $G_{1}$ and $G_{2}$ by

$$
G_{1}=\frac{1}{\sqrt{2}}(A-i \sqrt{\mu} B), \quad G_{2}=\frac{1}{\sqrt{2}}\left(A^{*}-i \sqrt{\mu} B^{*}\right) .
$$


Assume that $L \geq n$. Then the joint distribution of $G_{1}$ and $G_{2}$ is given by probability measure (2.1) with $a=\frac{1+\mu}{2 \mu}$ and $b=\frac{1-\mu}{2 \mu}$ for $0<\mu<1, m=2, V_{1}=V_{2}=0$, and $\nu_{1}=L-n$. The same model with $m=2$ and $V_{1}=V_{2}=0$ was generalised in [33, 3] to include non-trivial covariance matrices instead of the scalar parameters $a$ and $b$. Here, again only the squared singular values of the product matrix $Y_{2}$ were studied.

Without loss of generality we can always assume that the parameter $a$ in formula (2.1) can be set to $a=1$. Indeed, this can be achieved by defining new matrices $\widetilde{G_{1}}, \ldots, \widetilde{G_{m}}$ given by

$$
\widetilde{G_{l}}=\sqrt{a} G_{l}, \quad 1 \leq l \leq m,
$$

together with a replacement of the parameter $b$ by $b a^{-\frac{m}{2}}$, and a modification of the potential functions accordingly. From now on, we restrict our considerations to the probability measures defined by expression (2.1) with $a=1$.

\subsection{Density of product matrix processes and related biorthogonal ensembles.}

By definition, the density of the product matrix process corresponding to the multimatrix model (2.1) is that of the vector

$$
\underline{y}=\left(y^{m}, \ldots, y^{1}\right) \in\left(\mathbb{R}_{>0}^{n}\right)^{m},
$$

where $y^{l}$ is the vector of squared singular values of $G_{l} \cdots G_{1}$. Our first results gives the joint probability density of $\underline{y}$ explicitly.

Theorem 2.1. Consider a multi-matrix model defined by the probability measure (2.1), with $a=1$. Denote by $\left\{y_{j}^{l}\right\}_{j=1, \ldots, n}$ the set of squared singular values of the matrix $Y_{l}=G_{l} \cdots G_{1}$ for each $l=1, \ldots, m$. Thus for each $l$ the vector $y^{l}=\left(y_{1}^{l}, \ldots, y_{n}^{l}\right)$ contains the eigenvalues of the matrix $Y_{l}^{*} Y_{l}$. The joint probability density of $\underline{y}=\left(y^{m}, \ldots, y^{1}\right)$ reads

$$
\begin{aligned}
P_{n, m}(\underline{y})= & \frac{1}{Z_{n, m}} \operatorname{det}\left[\left(y_{j}^{m}\right)^{\frac{k-1}{2}} I_{k-1}\left(2 b\left(y_{j}^{m}\right)^{\frac{1}{2}}\right) e^{-V_{m}\left(y_{j}^{m}\right)}\right]_{j, k=1}^{n} \\
& \times \prod_{l=1}^{m-1} \operatorname{det}\left[\frac{\left(y_{j}^{l+1}\right)^{\nu_{l+1}}}{\left(y_{k}^{l}\right)^{\nu_{l+1}+1}} e^{-\frac{y_{j}^{l+1}}{y_{k}^{l}}-V_{l}\left(y_{k}^{l}\right)}\right]_{j, k=1}^{n} \operatorname{det}\left[\left(y_{j}^{1}\right)^{\nu_{1}+k-1} e^{-y_{j}^{1}}\right]_{j, k=1}^{n},
\end{aligned}
$$

where

$$
Z_{n, m}=(n !)^{m} \operatorname{det}\left[a_{i, j}\right]_{i, j=1}^{n},
$$

the matrix elements $a_{i, j}$ are given by

$$
\begin{aligned}
& a_{i, j}=\int_{0}^{\infty} \ldots \int_{0}^{\infty} t_{1}^{\nu_{1}+i-1}\left(\frac{t_{2}}{t_{1}}\right)^{\nu_{2}} \ldots\left(\frac{t_{m-1}}{t_{m-2}}\right)^{\nu_{m-1}}\left(\frac{t_{m}}{t_{m-1}}\right)^{\nu_{m}}\left(t_{m}\right)^{\frac{j-1}{2}} I_{j-1}\left(2 b\left(t_{m}\right)^{\frac{1}{2}}\right) \\
& \times e^{-t_{1}-\frac{t_{2}}{t_{1}}-\ldots-\frac{t_{m-1}}{t_{m-2}}-\frac{t_{m}}{t_{m-1}}-V_{1}\left(t_{1}\right)-V_{2}\left(t_{2}\right)-\ldots-V_{m-1}\left(t_{m-1}\right)-V_{m}\left(t_{m}\right)} \frac{d t_{1}}{t_{1}} \frac{d t_{2}}{t_{2}} \ldots \frac{d t_{m-1}}{t_{m-1}} d t_{m},
\end{aligned}
$$

and where $I_{\mu}(z)$ denotes the modified Bessel function of the first kind. 
Recall that the modified Bessel function of the first kind $I_{\mu}(z)$ is defined by

$$
I_{\mu}(z)=\sum_{m=0}^{\infty} \frac{1}{m ! \Gamma(\mu+m+1)}\left(\frac{z}{2}\right)^{2 m+\mu}
$$

and that the function $y^{\frac{\mu}{2}} I_{\mu}\left(2 b y^{\frac{1}{2}}\right)$ has the following asymptotic behaviour

$$
y^{\frac{\mu}{2}} I_{\mu}\left(2 b y^{\frac{1}{2}}\right) \sim \frac{(b y)^{\mu}}{\Gamma(\mu+1)}, \quad b \rightarrow 0 .
$$

From (2.3) and (2.7) we conclude that as $V_{1}=\ldots=V_{m}=0$, and as $b$ approaches zero, the joint probability density $P_{n, m}(\underline{y})$ becomes equal to

$$
\frac{1}{Z_{n, m}^{\text {Ginibre }}} \Delta_{n}\left(\left\{y_{j}^{m}\right\}\right) \prod_{l=1}^{m-1} \operatorname{det}\left[\frac{\left(y_{j}^{l+1}\right)^{\nu_{l+1}}}{\left(y_{k}^{l}\right)^{\nu_{l+1}+1}} e^{-\frac{y_{j}^{l+1}}{y_{k}^{l}}}\right]_{j, k=1}^{n} \operatorname{det}\left[\left(y_{j}^{1}\right)^{\nu_{1}+k-1} e^{-y_{j}^{1}}\right]_{j, k=1}^{n},
$$

where

$$
Z_{n, m}^{\text {Ginibre }}=(n !)^{m} \prod_{j=1}^{n} \prod_{l=0}^{m} \Gamma\left(j+\nu_{l}\right),
$$

and we have defined the Vandermonde determinant

$$
\Delta_{n}\left(\left\{y_{j}^{l}\right\}\right)=\operatorname{det}\left[\left(y_{j}^{l}\right)^{k-1}\right]_{j, k=1}^{n}=\prod_{1 \leq j<k \leq n}\left(y_{k}^{l}-y_{j}^{l}\right) .
$$

This is the density for the Ginibre product process, cf. Strahov [41, Proposition 4.2] (see also [5]). Note that the last determinant in (2.8) is proportional to the Vandermonde determinant $\Delta_{n}\left(\left\{y_{j}^{1}\right\}\right)$.

The vector $y^{m}=\left(y_{1}^{m}, \ldots, y_{n}^{m}\right)$ is that of the squared singular values of the total product matrix $Y_{m}=G_{m} \cdots G_{1}$. In view of the recent results on distributions of squared singular values for products of random matrices mentioned in the introduction, the probability distribution of $y^{m}$ is of special interest. Corollary 2.2 from Theorem 2.1 gives the joint probability distribution of $y^{m}$ explicitly.

Corollary 2.2. Consider the multi-matrix model defined by the probability distribution (2.1), and form the total product $Y_{m}=G_{m} \cdots G_{1}$. The squared singular values of $Y_{m}$ form a biorthogonal ensemble with the joint probability density function given by

$$
P_{n, m}\left(y_{1}^{m}, \ldots, y_{n}^{m}\right)=\frac{1}{Z_{n, m}^{\prime}} \operatorname{det}\left[\varphi_{i}\left(y_{j}^{m}\right)\right]_{i, j=1}^{n} \operatorname{det}\left[\psi_{i}\left(y_{j}^{m}\right)\right]_{i, j=1}^{n},
$$

with the normalisation $Z_{n, m}^{\prime}=n ! \operatorname{det}\left[a_{i, j}\right]_{i, j=1}^{n}$ resulting from (2.4). The functions $\varphi_{i}$, with $i=1, \ldots, n$ are given by

$$
\varphi_{i}(y)=y^{\frac{i-1}{2}} I_{i-1}\left(2 b y^{\frac{1}{2}}\right) e^{-V_{m}(y)}
$$


and the functions $\psi_{i}$ with $=1, \ldots, n$ are given by

$$
\begin{aligned}
\psi_{i}(y)= & \int_{0}^{\infty} \ldots \int_{0}^{\infty} t_{1}^{\nu_{1}+i-1}\left(\frac{t_{2}}{t_{1}}\right)^{\nu_{2}} \ldots\left(\frac{t_{m-1}}{t_{m-2}}\right)^{\nu_{m-1}}\left(\frac{y}{t_{m-1}}\right)^{\nu_{m}} \\
& \times e^{-t_{1}-\frac{t_{2}}{t_{1}}-\ldots-\frac{t_{m-1}}{t_{m-2}}-\frac{y}{t_{m-1}}-V_{1}\left(t_{1}\right)-V_{2}\left(t_{2}\right)-\ldots-V_{m-1}\left(t_{m-1}\right)} \frac{d t_{1}}{t_{1}} \frac{d t_{2}}{t_{2}} \ldots \frac{d t_{m-1}}{t_{m-1}}
\end{aligned}
$$

For some particular choices of potentials $V_{1}, \ldots, V_{m}$ we can obtain explicit expressions for the functions $\psi_{i}(y)$, and for the normalisation constant $Z_{n, m}^{\prime}$, as we will show below.

First, let us suppose that we could set all potentials $V_{1}=\ldots=V_{m}=0$. Then the functions $\psi_{i}(y)$ could be written as Meijer $G$-functions,

$$
\psi_{i}(y)=G_{0, m}^{m, 0}\left(\begin{array}{rrr}
- & \\
\nu_{1}+i-1, & \nu_{2}, & \ldots, \\
\nu_{m}
\end{array} \mid y\right) .
$$

For the resulting normalisation we would obtain from Andréief's integral identity

$$
Z_{n, m}^{\prime}=n ! \operatorname{det}\left[\int_{0}^{\infty} y^{\frac{i-1}{2}} I_{i-1}\left(2 b y^{\frac{1}{2}}\right) G_{0, m}^{m, 0}\left(\begin{array}{r}
- \\
\nu_{1}+j-1, \quad \nu_{2}, \quad \ldots, \quad \nu_{m}
\end{array} \mid y\right) d y\right]_{i, j=1}^{n} .
$$

However, for $m>2$ the integrals inside the determinant do not converge. The reason is that the modified Bessel function of the second kind (2.6) has the following asymptotic

$$
I_{i-1}\left(2 b y^{\frac{1}{2}}\right) \sim \frac{e^{2 b y^{\frac{1}{2}}}}{2 \pi^{\frac{1}{2}} b^{\frac{1}{2}} y^{\frac{1}{4}}}, y \rightarrow \infty,
$$

c.f. [38, which has to be compared to the asymptotic of the Meijer $G$-function [35]

$$
G_{0, m}^{m, 0}\left(\begin{array}{rl}
- & \\
\nu_{1}+j-1, & \nu_{2}, \quad \ldots, \quad \nu_{m}
\end{array} \mid y\right) \sim \frac{(2 \pi)^{\frac{m-1}{2}}}{m^{\frac{1}{2}}} y^{\theta} \exp \left[-m y^{\frac{1}{m}}\right], y \rightarrow \infty,
$$

with $\theta=\frac{1}{m}\left(\frac{1}{2}(1-m)+\nu_{1}+j-1+\sum_{l=2}^{m} \nu_{l}\right)$. Only for $m=2$ the two exponentials in eqs. (2.13) and (2.14) together lead to convergent integrals for $b<1$, and we obtain the biorthogonal ensemble equivalent to that studied by the authors in [7, 8] ]. Indeed for $m=2$ we have (recall that $\nu_{2}=0$ then)

$$
G_{0,2}^{2,0}\left(\begin{array}{cc|}
\nu_{1}+j-1, & 0
\end{array} \mid y\right)=2 y^{\frac{\nu_{1}+j-1}{2}} K_{\nu_{1}+j-1}\left(2 y^{\frac{1}{2}}\right)
$$

\footnotetext{
${ }^{1}$ We refer the reader to the book by Luke 35 for the definition of Meijer $G$-functions, and for their exact and asymptotic properties.

${ }^{2}$ For $m=1$ the corresponding one-matrix model is convergent for any $b$.
} 
where $K_{\kappa}(z)$ denotes the modified Bessel function of the second kind. It can be defined by the integral

$$
K_{\kappa}(z)=\frac{\Gamma\left(\kappa+\frac{1}{2}\right)(2 z)^{\kappa}}{\sqrt{\pi}} \int_{0}^{\infty} \frac{\cos (t) d t}{\left(t^{2}+z^{2}\right)^{\kappa+\frac{1}{2}}}, \quad \Re(\kappa)>-\frac{1}{2}
$$

which is more convenient for complex contour integrals over $\kappa$, or alternatively [38] as

$$
K_{\kappa}(2 z)=\frac{1}{2} z^{\kappa} \int_{0}^{\infty} d t t^{-\kappa-1} \exp \left[-t-\frac{z^{2}}{t}\right] .
$$

From the latter is is easy to obtain the following asymptotic expansion [38, 10.25.3], corresponding to (2.14) for $m=2$ :

$$
K_{\kappa}\left(2 b y^{\frac{1}{2}}\right) \sim \frac{\pi^{\frac{1}{2}}}{2^{\frac{1}{2}}\left(2 b y^{\frac{1}{2}}\right)^{\frac{1}{2}}} e^{-2 b y^{\frac{1}{2}}}, y \rightarrow \infty .
$$

It is not difficult to see that the model for $m=2$ studied in [7] in the parametrisation given in (2.2) can be mapped to our parametrisation (2.1), with $a=1$ and $b>0$ by rescaling in (2.2) $G_{1} \rightarrow \alpha_{1} G_{1}$ and $G_{2} \rightarrow \alpha_{2} G_{2}$, with $\alpha_{1}^{2}=\frac{2}{1+\mu}$ and $\alpha_{2}^{2}=\frac{1+\mu}{2 \mu}$, leading to $b=\frac{1-\mu}{\sqrt{4 \mu}}$. Therefore, in order to guarantee the existence of the corresponding matrix measure (2.1), for $m>2$ some non-zero potentials $V_{l}$ should be added for non-zero coupling $b>0$, to ensure convergence of the integrals.

In this paper the case corresponding to

$$
V_{1}(t)=\ldots=V_{m-2}(t)=0, \quad V_{m-1}(t)=b^{2} t, \quad V_{m}(t)=0,
$$

will be considered in detail $3^{3}$. Here, we are dealing with coupled matrices with the single coupling constant $b>0$. In this case, a simple contour integral representation for the correlation kernel suitable for an asymptotic analysis can be derived. Inserting the above conditions on the potentials (2.18) into the matrix measure (2.1), it turns into

$$
\begin{aligned}
& \frac{1}{Z_{n}(b)} e^{-\sum_{l=1}^{m} \operatorname{Tr}\left[G_{l}^{*} G_{l}\right]+b \operatorname{Tr}\left[G_{m} \cdots G_{1}+\left(G_{m} \cdots G_{1}\right)^{*}\right]-b^{2} \operatorname{Tr}\left[\left(G_{m-1} \cdots G_{1}\right)^{*}\left(G_{m-1} \cdots G_{1}\right)\right]} \prod_{l=1}^{m} d G_{l} \\
= & \frac{1}{Z_{n}(b)} e^{-\sum_{l=1}^{m-1} \operatorname{Tr}\left[G_{l}^{*} G_{l}\right]-\operatorname{Tr}\left[\left(G_{m}-b\left(G_{m-1} \cdots G_{1}\right)^{*}\right)\left(G_{m}^{*}-b G_{m-1} \cdots G_{1}\right)\right]} \prod_{l=1}^{m} d G_{l} .
\end{aligned}
$$

\footnotetext{
${ }^{3}$ Note that for $m=2$ the additional potential $V_{1}(t)=b t^{2}$ simply corresponds to a shift of the Gaussian term $\operatorname{Tr}\left[G_{1}^{*} G_{1}\right]$ which is already present.
} 
The normalised probability density of $\underline{y}=\left(y^{m}, \ldots, y^{1}\right)$ that follows from Theorem 2.1 by inserting (2.18) into (2.3) is given by

$$
\begin{aligned}
P_{n, m}(\underline{y} ; b)= & \frac{1}{Z_{n, m}(b)} \operatorname{det}\left[\left(y_{j}^{m}\right)^{\frac{k-1}{2}} I_{k-1}\left(2 b\left(y_{j}^{m}\right)^{\frac{1}{2}}\right)\right]_{j, k=1}^{n} \operatorname{det}\left[\frac{1}{y_{k}^{m-1}} e^{-\frac{y_{j}^{m}}{y_{k}^{m-1}-b^{2} y_{k}^{m-1}}}\right]_{j, k=1}^{n} \\
& \times \prod_{l=1}^{m-2} \operatorname{det}\left[\frac{\left(y_{j}^{l+1}\right)^{\nu_{l+1}}}{\left(y_{k}^{l}\right)^{\nu_{l+1}+1}} e^{-\frac{y_{j}^{l+1}}{y_{k}^{l}}}\right]_{j, k=1}^{n} \operatorname{det}\left[\left(y_{j}^{1}\right)^{\nu_{1}+k-1} e^{-y_{j}^{1}}\right]_{j, k=1}^{n},
\end{aligned}
$$

where the normalisation constant $Z_{n, m}(b)$ following from (2.4) remains to be determined, see (2.23) below. In what follows we will refer to the point process formed by random configuration $y$ with the joint probability density given by equation (2.20) as the Ginibre product process with coupling. The Ginibre product process with coupling is an oneparameter deformation of the Ginibre product process, and the coupling constant $b$ is the parameter of this deformation.

Remark 2.3. The model defined by equation (2.19) has the following interpretation. According to equation (2.19) the matrices $G_{1}, \ldots, G_{m}$ and $G_{m}^{\prime}=G_{m}-b\left(G_{m-1} \ldots G_{1}\right)^{*}$ can be understood as independent Ginibre matrices (each of the size $\left(n+\nu_{l}\right) \times\left(n+\nu_{l-1}\right)$, where $l=1, \ldots, m)$. Therefore, we will have the Ginibre product process on the first $m-1$ levels which will be independent of $b$. As $b \rightarrow 0$, the matrix $G_{m}-b\left(G_{m-1} \ldots G_{1}\right)^{*}$ turns into the Ginibre matrix $G_{m}$ independent of $G_{1}, \ldots, G_{m-1}$, and we obtain the Ginibre product process on all $m$ levels. As $b \rightarrow \infty$, the matrix $G_{m}^{\prime}$ is dominated by $\left(G_{m-1} \ldots G_{1}\right)^{*}$, so the singular values of $G_{m}^{\prime} G_{m-1} \ldots G_{1}$ will approach the squares of the singular values of $G_{m-1} \ldots G_{1}$.

Remark 2.4. If in the second line of (2.19) we rescale $G_{m} \rightarrow b G_{m}$ and then send $b \rightarrow \infty$ this leads to a delta function constraint, due to $\lim _{b \rightarrow \infty} b \exp \left[-b^{2}(x-y)^{2}\right] / \sqrt{\pi}=\delta(x-y)$, enforcing $G_{m}=\left(G_{m-1} \cdots G_{1}\right)^{*}$ at finite matrix sizes. It is precisely this limit that leads to the collapse of the Ginibre product process with coupling having $m$ levels to a Ginibre product process having only $m-1$ levels, as we will see below on the level of the joint probability density (2.20) for finite $n$, and in the large- $n$ limit in Theorem 3.2 (C).

It is instructive to consider separately the distribution of the squared singular values of the total product matrix $Y_{m}=G_{m} \cdots G_{1}$, in the case when the joint distribution of $G_{m}, \ldots, G_{1}$ is given by formula (2.19), with joint probability density (2.20). We obtain the following result.

Proposition 2.5. Consider the probability measure defined by equation (2.19), and let $Y_{m}=G_{m} \cdots G_{1}$ be the total product matrix of the corresponding multi-matrix model. The squared singular values $y_{1}^{m}, \ldots, y_{n}^{m}$ of $Y_{m}$ form a determinantal point process on $\mathbb{R}_{>0}$. This determinantal point process is a biorthogonal ensemble defined by the joint 
probability density $P\left(y_{1}^{m}, \ldots, y_{n}^{m} ; b\right)$. The explicit formula for $P\left(y_{1}^{m}, \ldots, y_{n}^{m} ; b\right)$ reads $(2.21)$

$$
P\left(y_{1}^{m}, \ldots, y_{n}^{m} ; b\right)=\frac{(n !)^{m-1}}{Z_{n, m}(b)} \operatorname{det}\left[\left(y_{j}^{m}\right)^{\frac{k-1}{2}} I_{k-1}\left(2 b\left(y_{j}^{m}\right)^{\frac{1}{2}}\right)\right]_{k, j=1}^{n} \operatorname{det}\left[\psi_{k}\left(y_{j}^{m}\right)\right]_{k, j=1}^{n},
$$

where

$$
\psi_{k}(y)=\frac{1}{2 \pi i} \int_{c-i \infty}^{c+i \infty} \prod_{l=2}^{m} \Gamma\left(u+\nu_{l}\right) \Gamma\left(u+\nu_{1}+k-1\right) \frac{2\left(b y^{\frac{1}{2}}\right)^{u} K_{u}\left(2 b y^{\frac{1}{2}}\right)}{\Gamma(u)} y^{-u} d u,
$$

with $c>0$ such that the poles of the Gamma-functions are to the right of the contour. The normalisation constant is given by

$$
Z_{n, m}(b)=(n !)^{m} b^{\frac{n(n-1)}{2}} \prod_{j=1}^{n} \prod_{l=1}^{m-1} \Gamma\left(j+\nu_{l}\right) .
$$

Note that following [38] we have

$$
\frac{2\left(b y^{\frac{1}{2}}\right)^{u} K_{u}\left(2 b y^{\frac{1}{2}}\right)}{\Gamma(u)} \sim 1, \quad b \rightarrow 0, \text { for } \Re(u)>0 .
$$

Taking this into account, and using the asymptotic expression $y^{\frac{j-1}{2}} I_{j-1}\left(2 b y^{\frac{1}{2}}\right)$ (see equation (2.7), contributing with an extra factor $1 / \Gamma(j))$, we find that the joint probability density function $P\left(y_{1}, \ldots, y_{n} ; b\right)$ defined by equation (2.21) has a limit as $b \rightarrow 0$. Namely, we have

$$
\begin{aligned}
& \lim _{b \rightarrow 0} P\left(y_{1}^{m}, \ldots, y_{n}^{m} ; b\right)=\frac{1}{n ! \prod_{i=1}^{n} \prod_{j=0}^{m} \Gamma\left(j+\nu_{j}\right)} \\
& \quad \times \Delta_{n}\left(\left\{y_{j}^{m}\right\}\right) \operatorname{det}\left[G_{0, m}^{m, 0}\left(\begin{array}{rrr}
\nu_{1}+k-1, & \nu_{2}, \quad \ldots, & \nu_{m}
\end{array} \mid y_{j}^{m}\right)\right]_{j, k=1}^{n} .
\end{aligned}
$$

This is the joint probability density function obtained by Akemann, Ipsen, and Kieburg [5] for the squared singular values of the ensemble of the products of $m$ independent rectangular matrices with independent complex Gaussian entries.

Now let us consider the behaviour of $P\left(y_{1}^{m}, \ldots, y_{n}^{m} ; b\right)$ as $b \rightarrow \infty$. Using the asymptotic formulae (2.13) and (2.17) of the modified Bessel functions involved, we find that as $b \rightarrow \infty$, the joint probability density function $P\left(y_{1}^{m}, \ldots, y_{n}^{m} ; b\right)$ defined by equation (2.21) becomes approximately equal to

$$
\begin{aligned}
& \sim \frac{1}{2^{n} b^{\frac{n(n+1)}{2}} n ! \prod_{i=1}^{n} \prod_{j=0}^{m-1} \Gamma\left(j+\nu_{j}\right) \prod_{j=1}^{n} y_{j}^{\frac{1}{2}}} \\
& \times \Delta_{n}\left(\left\{y_{j}^{m}\right\}\right) \operatorname{det}\left[G _ { 0 , m - 1 } ^ { m - 1 , 0 } \left(\begin{array}{cccc}
\nu_{1}+k-1, & \nu_{2}, & \ldots, & \left.\left.\nu_{m-1} \mid \frac{1}{b}\left(y_{j}^{m}\right)^{\frac{1}{2}}\right)\right]_{k, j=1}^{n} .
\end{array}\right.\right.
\end{aligned}
$$


Set $x_{j}=\frac{1}{b}\left(y_{j}^{m}\right)^{\frac{1}{2}}$. We then find that as $b \rightarrow \infty$, the joint probability density function of the new variables $x_{1}, \ldots, x_{n}$ converges to

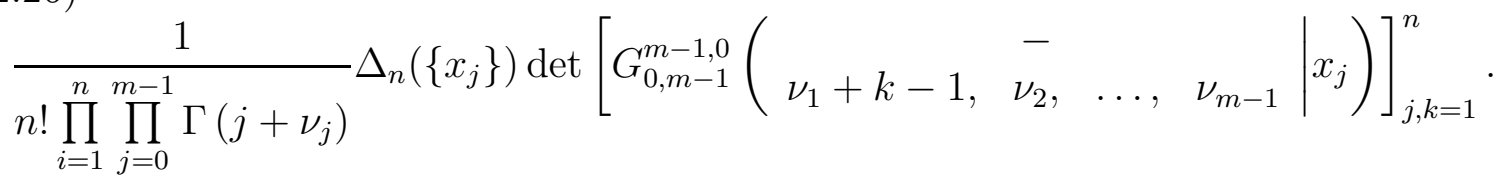

This is the joint probability density function for the ensemble of the squared singular values of the product of $m-1$ rectangular matrices with independent complex Gaussian entries. We conclude that the biorthogonal ensemble defined by equation (2.21) is an interpolating ensemble: it interpolates between the process of squared singular values from the ensemble of the products of $m$ independent rectangular matrices with independent complex Gaussian entries, and that of $m-1$ independent rectangular matrices with independent complex Gaussian entries.

As a final example we give the joint density of the product matrix process related to Hurwitz numbers via [9], as mentioned in the introduction. Compared to (2.1) it reads

$$
\frac{1}{Z_{n}} \exp \left[-\operatorname{Tr}\left[Q G_{1}^{*} G_{1}\right]-\sum_{l=2}^{m} \operatorname{Tr}\left[G_{l}^{*} G_{l}\right]-\operatorname{Tr}\left[V_{m}\left(\left(G_{m} \cdots G_{1}\right)^{*} G_{m} \cdots G_{1}\right)\right]\right] \prod_{l=1}^{m} d G_{l},
$$

that is $V_{1}=\ldots=V_{m-1}=0$ vanish, and in addition the Gaussian distribution of matrix $G_{1}$ now includes a nontrivial covariance matrix $Q$ of size $n \times n$ with positive eigenvalues $q_{1}, \ldots q_{n}>0$.

Proposition 2.6. Consider a multi-matrix model defined by probability measure (2.27). Denote by $\left\{y_{j}^{l}\right\}_{j=1, \ldots, m}$ the set of the squared singular values of the matrix $Y_{l}=G_{l} \ldots G_{1}$ for each $l=1, \ldots, m$. The joint probability density of $\underline{y}=\left(y^{m}, \ldots, y^{1}\right)$ is proportional to

$$
\operatorname{det}\left[\left(y_{j}^{m}\right)^{k-1} e^{-V_{m}\left(y_{j}^{m}\right)}\right]_{j, k=1}^{n} \prod_{l=1}^{m-1} \operatorname{det}\left[\frac{\left(y_{j}^{l+1}\right)^{\nu_{l+1}}}{\left(y_{k}^{l}\right)^{\nu_{l+1}+1}} e^{-\frac{y_{j}^{l+1}}{y_{k}^{l}}}\right]_{j, k=1}^{n} \operatorname{det}\left[\left(y_{j}^{1}\right)^{\nu_{1}} e^{-y_{j}^{1} q_{k}}\right]_{j, k=1}^{n} .
$$

The joint density of $y$ for the model defined by (2.27) can be obtained similarly to the proof of Theorem 2. $\overline{1}$, using the standard Harish-Chandra-Itzykson-Zuber formula. In the following we will not pursue this example further.

2.3. Exact formulae for the correlation kernels. Theorem 2.1 states that the density of the product matrix process associated with the multi-matrix model (2.1) can be written as a product of determinants. This enables us to apply the result by Eynard and Mehta [18], and to give a formula for all correlation functions. Namely, the Eynard-Mehta Theorem implies that the configuration of the squared singular values of all product matrices associated with the multi-matrix model (2.1) is a determinantal 
point process on $\{1, \ldots, m\} \times \mathbb{R}_{>0}$. The correlation kernel of this determinantal point process is given by the next theorem.

Theorem 2.7. Consider the multi-matrix model defined by the probability measure (2.1), with $a=1$. Denote by the set $\left\{y_{1}^{l}, \ldots, y_{n}^{l}\right\}$ the squared singular values of the matrix $Y_{l}=G_{l} \cdots G_{1}$, that are the eigenvalues of the matrix $Y_{l}^{*} Y_{l}$. The configuration of all these eigenvalues

$$
\left\{\left(l, y_{j}^{l}\right) \mid l=1, \ldots, m ; j=1, \ldots, n\right\}
$$

forms a determinantal point process on $\{1, \ldots, m\} \times \mathbb{R}_{>0}$. The correlation kernel of this determinantal point process, $K_{n, m}^{V}(r, x ; s, y)$, where $r, s \in\{1, \ldots, m\}$, and $x, y \in \mathbb{R}_{>0}$, can be written as

$$
K_{n, m}^{V}(r, x ; s, y)=-\phi_{r, s}(x, y)+\sum_{i, j=1}^{n} \phi_{r, m+1}(x, i)\left(A^{-1}\right)_{i, j} \phi_{0, s}(j, y) .
$$

Here, the elements of matrix $A=\left(a_{i, j}\right)$, with $i, j=1, \ldots, n$, are defined in (2.5). The kernel depends on three sets of functions which are given as follows.

(i) For the first set of functions $\phi_{r, s}(x, y)$ we distinguish the following cases:

- for $r=1, \ldots, m-1$ we have:

$$
\phi_{r, r+1}(x, y)=\left(\frac{y}{x}\right)^{\nu_{r+1}} \frac{e^{-\frac{y}{x}-V_{r}(x)}}{x} .
$$

- for $1 \leq r \leq m-2$ and $r+2 \leq s \leq m$ we have:

$$
\begin{aligned}
\phi_{r, s}(x, y)= & \frac{e^{-V_{r}(x)}}{x} \int_{0}^{\infty} \ldots \int_{0}^{\infty}\left(\frac{t_{r+1}}{x}\right)^{\nu_{r+1}}\left(\frac{t_{r+2}}{t_{r+1}}\right)^{\nu_{r+2}} \ldots\left(\frac{y}{t_{s-1}}\right)^{\nu_{s}} \\
& \times e^{-\frac{t_{r+1}}{x}-\frac{t_{r+2}}{t_{r+1}}-\ldots-\frac{t_{s-1}}{t_{s-2}}-\frac{y}{t_{s-1}}-V_{r+1}\left(t_{r+1}\right)-V_{r+2}\left(t_{r+2}\right) \ldots-V_{s-1}\left(t_{s-1}\right)} \\
& \times \frac{d t_{r+1}}{t_{r+1}} \frac{d t_{r+2}}{t_{r+2}} \ldots \frac{d t_{s-2}}{t_{s-2}} \frac{d t_{s-1}}{t_{s-1}} .
\end{aligned}
$$

- for $1 \leq s \leq r \leq m$ we have:

$$
\phi_{r, s}(x, y)=0
$$

(ii) For the second set of functions $\phi_{r, m+1}(x, j)$ we distinguish the following cases:

- for $1 \leq r \leq m-1$ we have :

$$
\begin{aligned}
\phi_{r, m+1}(x, j)= & \frac{e^{-V_{r}(x)}}{x} \int_{0}^{\infty} \ldots \int_{0}^{\infty}\left(\frac{t_{r+1}}{x}\right)^{\nu_{r+1}}\left(\frac{t_{r+2}}{t_{r+1}}\right)^{\nu_{r+2}} \ldots\left(\frac{t_{m}}{t_{m-1}}\right)^{\nu_{m}} t_{m}^{\frac{j-1}{2}} I_{j-1}\left(2 b t_{m}^{\frac{1}{2}}\right) \\
& \times e^{-\frac{t_{r+1}}{x}-\frac{t_{r+2}}{t_{r+1}}-\ldots-\frac{t_{m-1}}{t_{m-2}}-\frac{t_{m}}{t_{m-1}}-V_{r+1}\left(t_{r+1}\right)-V_{r+2}\left(t_{r+2}\right) \ldots-V_{m-1}\left(t_{m-1}\right)-V_{m}\left(t_{m}\right)} \\
& \times \frac{d t_{r+1}}{t_{r+1}} \frac{d t_{r+2}}{t_{r+2}} \ldots \frac{d t_{m-1}}{t_{m-1}} d t_{m} .
\end{aligned}
$$


- for $r=m$ we have:

$$
\phi_{m, m+1}(x, j)=x^{\frac{j-1}{2}} I_{j-1}\left(2 b x^{\frac{1}{2}}\right) e^{-V_{m}(x)} .
$$

(iii) For the third set of functions $\phi_{0, s}(i, y)$ we distinguish the following cases:

- for $2 \leq s \leq m$ we have:

$$
\begin{aligned}
\phi_{0, s}(i, y)= & \int_{0}^{\infty} \ldots \int_{0}^{\infty} t_{1}^{\nu_{1}+i-1}\left(\frac{t_{2}}{t_{1}}\right)^{\nu_{2}} \ldots\left(\frac{t_{s-1}}{t_{s-2}}\right)^{\nu_{s-1}}\left(\frac{y}{t_{s-1}}\right)^{\nu_{s}} \\
& \times e^{-t_{1}-\frac{t_{2}}{t_{1}}-\ldots-\frac{t_{s-1}}{t_{s-2}}-\frac{y}{t_{s-1}}-V_{1}\left(t_{1}\right)-V_{2}\left(t_{2}\right)-\ldots-V_{s-2}\left(t_{s-2}\right)-V_{s-1}\left(t_{s-1}\right)} \\
& \times \frac{d t_{1}}{t_{1}} \frac{d t_{2}}{t_{2}} \ldots \frac{d t_{s-2}}{t_{s-2}} \frac{d t_{s-1}}{t_{s-1}}
\end{aligned}
$$

- for $s=1$ we have:

$$
\phi_{0,1}(i, y)=y^{\nu_{1}+i-1} e^{-y}
$$

Recall that the case corresponding to $V_{1}(t)=\ldots=V_{m-2}(t)=0, V_{m-1}(t)=b^{2} t$, and $V_{m}(t)=0$ corresponds to the Ginibre product process with coupling. Let us denote the relevant correlation kernel by $K_{n, m}(r, x ; s, y ; b)$, to emphasise its dependence on the coupling constant $b$. For the Ginibre product process with coupling we are able to reduce all multiple integrals in the statement of Theorem 2.7 to at most single integrals, expressed in terms of special functions, and to find the matrix $A^{-1}$ explicitly. This leads to a contour integral representation for the correlation kernel $K_{n, m}(r, x ; s, y ; b)$ as follows.

Theorem 2.8. For the special case $V_{1}(t)=\ldots=V_{m-2}(t)=0, V_{m-1}(t)=b^{2} t$, and $V_{m}(t)=0$ the correlation kernel of Theorem 2.7 can be written as

$$
K_{n, m}(r, x ; s, y ; b)=-\phi_{r, s}(x, y ; b)+S_{n, m}(r, x ; s, y ; b) .
$$

The functions $\phi_{r, s}(x, y ; b)$ are given by

$$
\begin{aligned}
& \phi_{r, s}(x, y ; b)
\end{aligned}
$$

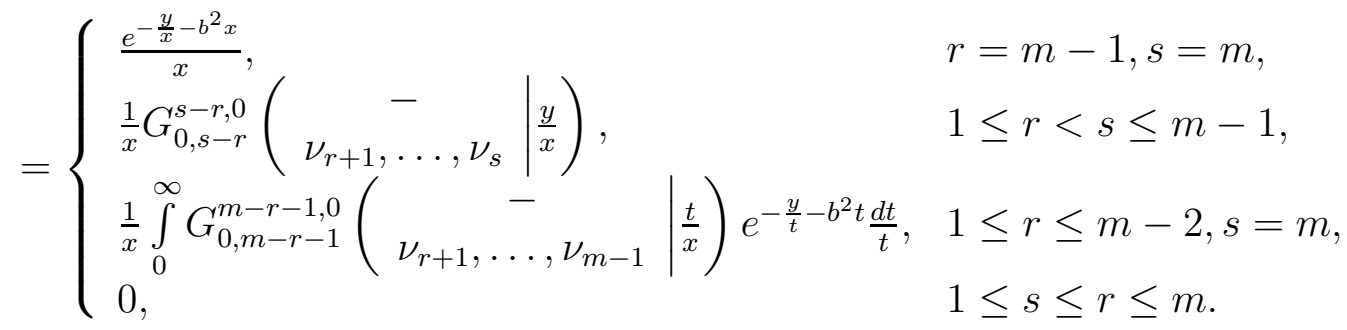


The functions $S_{n, m}(r, x ; s, y ; b)$ can be written as

$$
\begin{aligned}
S_{n, m}(r, x ; s, y ; b)= & \frac{1}{(2 \pi i)^{2}} \int_{-\frac{1}{2}-i \infty}^{-\frac{1}{2}+i \infty} d u \oint_{\Sigma_{n}} d t \frac{\Gamma(t-n+1)}{\Gamma(u-n+1)} \frac{\prod_{j=0}^{s} \Gamma\left(u+\nu_{j}+1\right)}{\prod_{j=0}^{r} \Gamma\left(t+\nu_{j}+1\right)} \\
& \times \frac{x^{t} p_{r}(t, x ; b) y^{-u-1} q_{s}(u+1, y ; b)}{u-t} .
\end{aligned}
$$

Here, $\Sigma_{n}$ is a closed contour encircling $0,1, \ldots, n$ in positive direction and such that $\operatorname{Re} t>-\frac{1}{2}$ for $t \in \Sigma_{n}$. In (2.39) the functions $p_{r}(t, x ; b)$ are defined by

$$
p_{r}(t, x ; b)= \begin{cases}1, & r \in\{1, \ldots, m-1\}, \\ \frac{\Gamma(t+1) I_{t}\left(2 b x^{\frac{1}{2}}\right)}{\left(b x^{\frac{1}{2}}\right)^{t}}, & r=m,\end{cases}
$$

and the functions $q_{s}(u, y ; b)$ are defined by

$$
q_{s}(u, y ; b)= \begin{cases}1, & s \in\{1, \ldots, m-1\}, \\ \frac{2\left(b y^{\frac{1}{2}}\right)^{u} K_{u}\left(2 b y^{\frac{1}{2}}\right)}{\Gamma(u)}, & s=m .\end{cases}
$$

Remarks. (a) As a determinantal point process between the first $m-1$ levels, that is on $\{1, \ldots, m-1\} \times \mathbb{R}_{>0}$, the kernel (2.37) is independent of $b$ and agrees with kernel $K_{n, m-1}^{\text {Ginibre }}(r, x ; s, y)$ of the Ginibre product process without coupling found by Strahov [41, Prop.2.3]:

$$
\begin{aligned}
K_{n, m-1}^{\text {Ginibre }}(r, x ; s, y)= & -\frac{1}{x} G_{0, s-r}^{s-r, 0}\left(\begin{array}{c}
- \\
\nu_{r+1}, \ldots, \nu_{s}
\end{array} \frac{y}{x}\right) \mathbf{1}_{s>r} \\
& +\frac{1}{(2 \pi i)^{2}} \int_{-\frac{1}{2}-i \infty}^{-\frac{1}{2}+i \infty} d u \oint_{\Sigma_{n}} d t \frac{\prod_{j=0}^{s} \Gamma\left(u+\nu_{j}+1\right)}{\prod_{j=0}^{r} \Gamma\left(t+\nu_{j}+1\right)} \frac{\Gamma(t-n+1)}{\Gamma(u-n+1)} \frac{x^{t} y^{-u-1}}{u-t},
\end{aligned}
$$

where $\mathbf{1}_{s>r}$ is the indicator function ${ }^{4}$. Because this agreement holds at finite matrix size $n$ it will also hold for the limiting kernel. For $r=m$ or $s=m$ the kernel $K_{n, m}(r, x ; s, y ; b)$ (2.37) depends on $b$ and differs from the kernel (2.42) for the Ginibre product process.

(b) As $b \rightarrow 0$, also for $r=m$ or $s=m$ the correlation kernel $K_{n, m}(r, x ; s, y ; b)$ turns into the correlation kernel $K_{n, m}^{\text {Ginibre }}(r, x ; s, y)$ of the Ginibre product process (2.42) (with $m-1 \rightarrow m)$ from [41, Prop.2.3].

As a particular case (corresponding to $r=s=m$ ) we obtain from Theorem 2.8 a double contour integral representation for the correlation kernel of the biorthogonal ensemble defined in Proposition 2.5, where only the squared singular values of the total product matrix $Y_{m}$ are retained.

\footnotetext{
${ }^{4}$ We follow the convention here that the index $m-1$ of the kernel on the left-hand side indicates the range that the arguments $r$ and $s$ can take.
} 
Proposition 2.9. Consider the biorthogonal ensemble of Proposition 2.5. This biorthogonal ensemble defines a determinantal point process on $\mathbb{R}_{>0}$. The correlation kernel of this determinantal point process can be written as

$$
\begin{aligned}
K_{n, m}(x ; y ; b)= & \frac{1}{(2 \pi i)^{2}} \int_{-\frac{1}{2}-i \infty}^{-\frac{1}{2}+i \infty} d u \oint_{\Sigma_{n}} d t \frac{\Gamma(t-n+1)}{\Gamma(u-n+1)} \frac{\prod_{j=0}^{m} \Gamma\left(u+\nu_{j}+1\right)}{\prod_{j=0}^{m} \Gamma\left(t+\nu_{j}+1\right)} \\
& \times \frac{x^{t} y^{-u-1}}{u-t} \frac{2 \Gamma(t+1)\left(b y^{\frac{1}{2}}\right)^{u+1}}{\Gamma(u+1)\left(b x^{\frac{1}{2}}\right)^{t}} I_{t}\left(2 b x^{\frac{1}{2}}\right) K_{u+1}\left(2 b y^{\frac{1}{2}}\right) .
\end{aligned}
$$

As $b \rightarrow 0$, due to eqs. (2.7) and (2.24) the last factors in the second line of (2.43) can be replaced by 1 , and we obtain

$$
\lim _{b \rightarrow 0} K_{n, m}(x, y ; b)=\frac{1}{(2 \pi i)^{2}} \int_{-\frac{1}{2}-i \infty}^{-\frac{1}{2}+i \infty} d u \oint_{\Sigma_{n}} d t \frac{\Gamma(t-n+1)}{\Gamma(u-n+1)} \frac{\prod_{j=0}^{m} \Gamma\left(u+\nu_{j}+1\right)}{\prod_{j=0}^{m} \Gamma\left(t+\nu_{j}+1\right)} \frac{x^{t} y^{-u-1}}{u-t} .
$$

The expression in the right-hand side of this equation represents the correlation kernel for the ensemble of the squared singular values of $m$ rectangular matrices with independent complex Gaussian entries, see Kuijlaars and Zhang [31, Prop.5.1].

2.4. Hierarchy of correlation kernels at finite matrix size. In order to summarise the results presented above let us briefly describe the hierarchy of the correlation kernels under considerations. On top of the hierarchy we have the correlation kernel $K_{n, m}^{V}(r, x ; s, y)$ (2.29) of Theorem 2.7. This kernel depends on $m$ potential functions $V_{1}(t), \ldots, V_{m}(t)$, that couple the different levels, and lives on the space $\{1, \ldots, m\} \times \mathbb{R}_{>0}$.

The correlation kernel $K_{n, m}(r, x ; s, y ; b)$ (2.37) of Theorem 2.8 is the specialisation of $K_{n, m}^{V}(r, x ; s, y)$ to the case where $V_{1}(t)=\ldots=V_{m-2}(t)=0, V_{m-1}(t)=b^{2} t$, and $V_{m}(t)=0$. It depends on one coupling constant $b>0$, and defines the Ginibre product process with coupling. As we pointed out already, due to our choice of potentials this point process agrees with the Ginibre product process without coupling for the first $m-1$ levels, with $r, s<m$. On the other hand, taking $r=s=m$, we obtain the kernel $K_{n, m}(x, y ; b)$ that depends on the parameter $b$. It describes the biorthogonal ensemble for the singular values of the total product matrix with coupling. Thus, for $r=m$ or $s=m$ the kernel $K_{n, m}(r, x ; s, y ; b)$ coupled to the $m$ th level can be understood as a deformation of the kernel $K_{n, m}^{\text {Ginibre }}(r, x ; s, y)$ of the Ginibre product process. For $r=s=m$, the latter kernel $K_{n, m}^{\text {Ginibre }}(m, x ; m, y)$ specialises to the finite- $n$ Meijer $G$ kernel, which is the correlation kernel for the ensemble of the squared singular values of $m$ rectangular matrices with independent complex Gaussian entries. Note that the finite$n$ Meijer $G$-kernel can be obtained from $K_{n, m}(x, y ; b)$ by taking the coupling parameter 
$b$ to zero. Finally, if $r=s=1$, the kernel $K_{n, m}^{\text {Ginibre }}(1, x ; 1, y)$ specialises to that of the classical Laguerre ensemble.

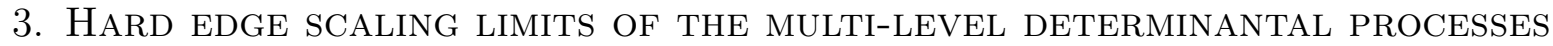

The point processes considered in this paper are uniquely determined by their correlation functions. Therefore, we will say that the point processes $\mathcal{P}$ converges to the point process $\mathcal{P}^{\prime}$, if all correlation functions of $\mathcal{P}$ converge to the corresponding correlation functions of $\mathcal{P}^{\prime}$. Since we are dealing with determinantal point processes only, the convergence of the correlation kernel of $\mathcal{P}$ to the correlation kernel of $\mathcal{P}^{\prime}$ will be considered as equivalent to the convergence of $\mathcal{P}$ to $\mathcal{P}^{\prime}$.

In order to discuss scaling limits of the multi-level determinantal processes it is convenient to introduce the following notation, following [41]. Denote by $K_{\infty, m}^{\text {Ginibre }}(r, x ; s, y)$ the kernel given by the formula

$$
\begin{aligned}
K_{\infty, m}^{\text {Ginibre }}(r, x ; s, y)= & -\frac{1}{x} G_{0, s-r}^{s-r, 0}\left(\begin{array}{c}
- \\
\nu_{r+1}, \ldots, \nu_{s}
\end{array} \mid \frac{y}{x}\right) \mathbf{1}_{s>r} \\
& +\frac{1}{(2 \pi i)^{2}} \int_{-\frac{1}{2}-i \infty}^{-\frac{1}{2}+i \infty} d u \oint_{\Sigma_{\infty}} d t \frac{\prod_{j=0}^{s} \Gamma\left(u+\nu_{j}+1\right)}{\prod_{j=0}^{r} \Gamma\left(t+\nu_{j}+1\right)} \frac{\sin \pi u}{\sin \pi t} \frac{x^{t} y^{-u-1}}{u-t} .
\end{aligned}
$$

Here $x>0, y>0$, the parameters $r, s$ take values in $\{1, \ldots, m\}$, and $\Sigma_{\infty}$ is a contour starting from $+\infty$ in the upper half plane and returning to $+\infty$ in the lower half plane, leaving $-\frac{1}{2}$ on the left, and encircling $\{0,1,2, \ldots\}$. Here, we write the correlation kernel $K_{\infty, m}^{\text {Ginibre }}(r, x ; s, y)$ with an index $m$, only to emphasise that the variables $r$ and $s$ take values in $\{1, \ldots, m\}$. Note that for $r=s$ the kernel $K_{\infty, m}^{\text {Ginibre }}(r, x ; r, y)=K_{\infty, r}^{\text {Ginibre }}(r, x ; r, y)$ is the limiting Meijer G-kernel obtained by Kuijlaars and Zhang [31]. It describes the hard edge scaling limit for the product of $r$ independent complex Gaussian matrices and reduces to the standard Bessel kernel for $r=1$. In [41] Strahov showed that $K_{\infty, m}^{\text {Ginibre }}(r, x ; s, y)$ can be understood as a multi-level extension of the infinite Meijer $G$-kernel.

In the context of products of random matrices, the kernel $K_{\infty, m}^{\text {Ginibre }}(r, x ; s, y)$ describes the hard edge scaling limit of the Ginibre product process. Recall that the Ginibre product process is a determinantal point process on $\{1, \ldots, m\} \times \mathbb{R}_{>0}$ whose density is proportional to expression (2.8), and whose correlation kernel is given by formula (2.42). The following proposition was shown in [41]:

Proposition 3.1. Assume that the point configurations $\left(y_{1}^{m}, \ldots, y_{n}^{m} ; \ldots ; y_{1}^{1}, \ldots, y_{n}^{1}\right)$ form the Ginibre product process on $\{1, \ldots, m\} \times \mathbb{R}_{>0}$, given by (2.8). As $n \rightarrow \infty$, the scaled Ginibre process formed by the point configurations $\left(n y_{1}^{m}, \ldots, n y_{n}^{m} ; \ldots ; n y_{1}^{1}, \ldots, n y_{n}^{1}\right)$ converges to the determinantal point process $\mathcal{P}_{\infty, m}^{\text {Ginibre }}$ on $\{1, \ldots, m\} \times \mathbb{R}_{>0}$, whose correlation kernel, $K_{\infty, m}^{\text {Ginibre }}(r, x ; s, y)$, is defined by equation (3.1). 
Alternatively, we have

$$
\lim _{n \rightarrow \infty} \frac{1}{n} K_{n, m}^{\text {Ginibre }}\left(r, \frac{x}{n} ; s, \frac{y}{n}\right)=K_{\infty, m}^{\text {Ginibre }}(r, x ; s, y),
$$

where $K_{n, m}^{\text {Ginibre }}(r, x ; s, y)$ is defined by equation 2.42) and $K_{\infty, m}^{\text {Ginibre }}(r, x ; s, y)$ by equation (3.1). The variables $x$ and $y$ take values in a compact subset of $\mathbb{R}_{>0}$, and $1 \leq r, s \leq m$.

Now, let us consider the convergence of the Ginibre product process with coupling. Recall that by this we mean the multi-level determinantal process on $\{1, \ldots, m\} \times \mathbb{R}_{>0}$ whose density is given by expression (2.20) and whose correlation kernel $K_{n, m}(r, x ; s, y ; b)$ is given by Theorem 2.8. As we already pointed out previously, for $r, s \leq m-1$ this kernel agrees with that of the Ginibre product process without coupling, (2.42), for which the hard edge limit was already worked out in [41, see (3.2) in Proposition 3.1 above (with $m-1 \rightarrow m)$. Therefore, in the following theorem we will only find nontrivial results for the limit of the kernel $K_{n, m}(r, x ; s, y ; b)$ with $r=m$ or $s=m$, that includes the $m$-th level. In particular we will now drop the assumption that the coupling parameter $b$ is constant, and consider $b$ as a function of the number of particles on the same level, $b=b(n)$. Recall that once $b$ approaches zero, the Ginibre product process with coupling turns into that without, as discussed in Section 2. Depending on how $b(n)$ behaves as a function of $n$, we find three different limits for the limiting kernel that includes level $m$.

Theorem 3.2. Let the point configurations $\left(y_{1}^{m}, \ldots, y_{n}^{m} ; \ldots ; y_{1}^{1}, \ldots, y_{n}^{1}\right)$ with density (2.20) form the Ginibre product process with coupling on $\{1, \ldots, m\} \times \mathbb{R}_{>0}$, with correlation kernel $K_{n, m}(r, x ; s, y ; b)$ of Theorem 2.8, equation (2.37).

(A) Weak coupling regime. Assume that $b(n) / \sqrt{n} \rightarrow 0$, as $n \rightarrow \infty$. The scaled Ginibre product process with coupling of configurations $\left(n y_{1}^{m}, \ldots, n y_{n}^{m} ; \ldots ; n y_{1}^{1}, \ldots, n y_{n}^{1}\right)$ converges as $n \rightarrow \infty$ to the determinantal point process $\mathcal{P}_{\infty, m}^{\text {Ginibre }}$. Equivalently, we have the following relation to the limiting kernel of the Ginibre product process (3.1), for all levels $1 \leq r, s \leq m$ :

$$
\lim _{n \rightarrow \infty} \frac{1}{n} K_{n, m}\left(r, \frac{x}{n} ; s, \frac{y}{n} ; b(n)\right)=K_{\infty, m}^{\text {Ginibre }}(r, x ; s, y) .
$$

Here, the variables $x$ and $y$ take values in a compact subset of $\mathbb{R}_{>0}$.

(B) Interpolating regime. Assume that $b(n) / \sqrt{n} \rightarrow \alpha$, as $n \rightarrow \infty$, where $\alpha>0$. As $n \rightarrow \infty$, the scaled Ginibre product process with coupling formed by the point configurations $\left(n y_{1}^{m}, \ldots, n y_{n}^{m} ; \ldots ; n y_{1}^{1}, \ldots, n y_{n}^{1}\right)$ converges to the determinantal point process $\mathcal{P}_{\infty, m}^{\text {interpol }}(\alpha)$ on $\{1, \ldots, m\} \times \mathbb{R}_{>0}$. Its correlation kernel is given by

$$
\begin{aligned}
& K_{\infty, m}^{\text {interpol }}(r, x ; s, y ; \alpha)=-\phi_{r, s}(x, y ; \alpha) \\
& +\frac{1}{(2 \pi i)^{2}} \int_{-\frac{1}{2}-i \infty}^{-\frac{1}{2}+i \infty} d u \oint_{\Sigma_{\infty}} d t \frac{\prod_{j=0}^{s} \Gamma\left(u+\nu_{j}+1\right)}{\prod_{j=0}^{r} \Gamma\left(t+\nu_{j}+1\right)} \frac{\sin \pi u}{\sin \pi t} \frac{x^{t} p_{r}(t, x ; \alpha) q_{s}(u+1, y ; \alpha)}{y^{u+1}(u-t)},
\end{aligned}
$$

for $r=m$ or $s=m$, with the functions $\phi_{r, s}(x, y ; \alpha), p_{r}(t, x ; \alpha)$, and $q_{s}(u+1, y ; \alpha)$ defined by equations (2.38), 2.40), and (2.41), respectively. For $1 \leq r, s \leq m-1$ its correlation 
kernel is given by $K_{\infty, m-1}^{\text {Ginibre }}(r, x ; s, y)$. Equivalently, we have for $r=m$ or $s=m$ :

$$
\lim _{n \rightarrow \infty} \frac{1}{n} K_{n, m}\left(r, \frac{x}{n} ; s, \frac{y}{n} ; b(n)\right)=K_{\infty, m}^{\text {interpol }}(r, x ; s, y ; \alpha),
$$

and (3.3) (with $m \rightarrow m-1$ on the right-hand side) for $1 \leq r, s \leq m-1$. Here, the variables $x$ and $y$ take values in a compact subset of $\mathbb{R}_{>0}$.

(C) Strong coupling regime. Assume that $b(n) / \sqrt{n} \rightarrow \infty$, as $n \rightarrow \infty$. The scaled process Ginibre product process with coupling formed by the point configurations

$$
\left(\frac{n}{b(n)}\left(y_{1}^{m}\right)^{\frac{1}{2}}, \ldots, \frac{n}{b(n)}\left(y_{n}^{m}\right)^{\frac{1}{2}} ; n y_{1}^{m-1}, \ldots, n y_{n}^{m-1} ; \ldots ; n y_{1}^{1}, \ldots, n y_{n}^{1}\right)
$$

converges as $n \rightarrow \infty$ to the determinantal point process $\mathcal{P}_{\infty, m-1}^{\text {Ginibre }}$ of $m-1$ levels, with the following identification of levels $m-1$ and $m$ on $\{1, \ldots, m\} \times \mathbb{R}_{>0}$. Namely, its correlation kernel is given by the following limiting relations:

- For $1 \leq r, s \leq m-1$ we have equation (3.3) (with $m \rightarrow m-1$ on the right-hand side).

- For $1 \leq r \leq m-1, s=m$ we have

$$
\begin{aligned}
& \lim _{n \rightarrow \infty} \frac{2^{\frac{1}{2}} b(n) y^{\frac{1}{2}}}{n^{\frac{3}{2}}} K_{n, m}\left(r, \frac{x}{n} ; m, \frac{b^{2}(n) y^{2}}{n^{2}} ; b(n)\right) \frac{e^{2 \frac{b^{2}(n)}{n} y}}{2^{\frac{1}{2}} \pi^{\frac{1}{2}}} \\
& =K_{\infty, m-1}^{\text {Ginibre }}(r, x ; m-1, y)+\mathbf{1}_{r, m-1} \delta(x-y) .
\end{aligned}
$$

- For $r=m, 1 \leq s \leq m-1$ we have

$$
\lim _{n \rightarrow \infty} \frac{2^{\frac{1}{2}} b(n) x^{\frac{1}{2}}}{n^{\frac{3}{2}}} K_{n, m}\left(m, \frac{b^{2}(n) x^{2}}{n^{2}} ; s, \frac{y}{n} ; b(n)\right) \frac{2^{\frac{1}{2}} \pi^{\frac{1}{2}}}{e^{2 \frac{b^{2}(n)}{n} x}}=K_{\infty, m-1}^{\text {Ginibre }}(m-1, x ; s, y) .
$$

- For $r=m$ and $s=m$ we have

$$
\begin{aligned}
& \lim _{n \rightarrow \infty} \frac{2 b^{2}(n) x^{\frac{1}{2}} y^{\frac{1}{2}}}{n^{2}} K_{n, m}\left(m, \frac{b^{2}(n) x^{2}}{n^{2}} ; m, \frac{b^{2}(n) y^{2}}{n^{2}} ; b(n)\right) \frac{e^{2 \frac{b^{2}(n)}{n} y}}{e^{\frac{b^{2}(n)}{n}} x} \\
& =K_{\infty, m-1}^{\text {Ginibre }}(m-1, x ; m-1, y) .
\end{aligned}
$$

In all these formulas the variables $x$ and $y$ take values in a compact subset of $\mathbb{R}_{>0}$.

Remarks. (a) The interpolating point process $\mathcal{P}_{\infty, m}^{\text {interpol }}(\alpha)$, that contains the correlation kernel $K_{\infty, m}^{\text {interpol }}(r, x ; s, y ; \alpha)$ for $r$ or $s=m$, can be understood as a one-parameter deformation of the determinantal point process $\mathcal{P}_{\infty, m}^{\text {Ginibre }}$ defined by the correlation kernel $K_{\infty, m}^{\text {Ginibre }}(r, x ; s, y)$. This will be made more precise in the next Section 4 .

(b) The limiting point process obtained in the strong coupling regime is a determinantal process living on $m-1$ levels, with an infinite number of points on each level, and where the levels $m$ and $m-1$ have been identified. The correlations between particles on the first $m-1$ levels trivially agrees with that of $\mathcal{P}_{\infty, m-1}^{\text {Ginibre }}$, the hard edge scaling limit of the Ginibre product process with $m-1$ levels. The correlations between particles only on the $m$-th level agree with those of the Ginibre product process on the $(m-1)$-th level. Furthermore, the correlations between particles on the $m$-th level and all other particles on the first $m-1$ levels are the same as if all the particles on the $m$-th level would be on 
the $(m-1)$-th level - unless two points on level $m$ and level $m-1$ coincide, hence the contact interaction in terms of the Dirac delta. In other words, in the asymptotic strong coupling regime the point process on the $m$-th level collapses to that on the $(m-1)$-th level, and the $m$-matrix model behaves statistically like an $(m-1)$-matrix model.

(c) In particular, for $m=2$ the limiting process at strong coupling collapses to the classical Bessel-kernel process of a single level, with the collapse of the first to the second level described in the previous remark.

For the biorthogonal ensemble formed by the singular values of the total product matrix $Y_{m}=G_{m} \cdots G_{1}$ only, Theorem 3.2 leads to the following result.

Corollary 3.3. Let $Y_{m}=G_{m} \cdots G_{1}$ be the total product matrix of the multi-matrix model defined by equation (2.19), and let $\left(y_{1}, \ldots, y_{n}\right)$ be the squared singular values of $Y_{m}$.

(A) Assume that $b(n) / \sqrt{n} \rightarrow 0$, as $n \rightarrow \infty$. Then, the point process formed by the point configurations $\left(n y_{1}, \ldots, n y_{n}\right)$ converges to the process for the product of $m$ independent Gaussian matrices, i.e. to the determinantal process on $\mathbb{R}_{>0}$ with the correlation kernel given by the Meijer $G$-kernel $K_{\infty, m}^{\text {Ginibre }}(m, x ; m, y)$, equation (3.1).

(B) Assume that $b(n) / \sqrt{n} \rightarrow \alpha$, as $n \rightarrow \infty$, where $\alpha>0$. Then, the point process formed by the point configurations $\left(n y_{1}, \ldots, n y_{n}\right)$ converges to the determinantal process on $\mathbb{R}_{>0}$ defined by the correlation kernel $K_{\infty, m}^{\text {interpol }}(m, x ; m, y ; \alpha)$, given by equation (3.4). The limiting determinantal process can be understood as a one-parameter deformation of the Meijer G-kernel process for the product of $m$ independent Gaussian matrices.

(C) Assume that $b(n) / \sqrt{n} \rightarrow \infty$, as $n \rightarrow \infty$. Then, the scaled process formed by the point configurations $\left(\frac{n}{b(n)}\left(y_{1}^{m}\right)^{\frac{1}{2}}, \ldots, \frac{n}{b(n)}\left(y_{n}^{m}\right)^{\frac{1}{2}}\right)$ converges to the determinantal process on $\mathbb{R}_{>0}$ with the correlation kernel given by the Meijer $G$-kernel $K_{\infty, m-1}^{\text {Ginibre }}(m-1, x ; m-1, y)$, the limiting process for the product of $m-1$ independent Gaussian matrices.

\section{The InTERPolating MUlti-LEVEL DeterminANTAL PROCESS}

Consider the determinantal process $\mathcal{P}_{\infty, m}^{\text {interpol }}(\alpha)$ on $\{1, \ldots, m\} \times \mathbb{R}_{>0}$ in the interpolating regime. We will show that it interpolates between the hard edge scaling limit $\mathcal{P}_{\infty, m}^{\text {Ginibre }}$ of the Ginibre product process of $m$ independent levels, and that in the strong coupling regime among $m-1$ levels. For this reason we will refer to $\mathcal{P}_{\infty, m}^{\text {interpol }}(\alpha)$ as to the interpolating multi-level determinantal process.

Theorem 4.1. Assume that the point configurations

$$
\left(x_{1}^{m}, x_{2}^{m}, \ldots ; x_{1}^{m-1}, x_{2}^{m-1}, \ldots ; \ldots ; x_{1}^{1}, x_{2}^{1}, \ldots\right)
$$

form the determinantal process $\mathcal{P}_{\infty, m}^{\text {interpol }}(\alpha)$ on $\{1, \ldots, m\} \times \mathbb{R}_{>0}$ in the interpolating regime.

(A) As $\alpha \rightarrow 0$, this determinantal process $\mathcal{P}_{\infty, m}^{\text {interpol }}(\alpha)$ converges to the determinan-

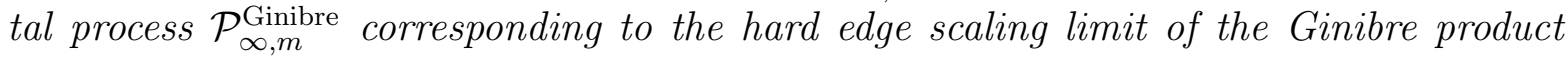
process of $m$ levels. In particular, for $r=m$ or $s=m$ we find

$$
\lim _{\alpha \rightarrow 0} K_{\infty, m}^{\text {interpol }}(r, x ; s, y ; \alpha)=K_{\infty, m}^{\text {Ginibre }}(r, x ; s, y) .
$$


where the variables $x$ and $y$ take values in a compact subset of $\mathbb{R}_{>0}$.

(B) Consider the scaled interpolating process formed by the point configurations

$$
\left(\frac{1}{\alpha}\left(x_{1}^{m}\right)^{\frac{1}{2}}, \frac{1}{\alpha}\left(x_{2}^{m}\right)^{\frac{1}{2}}, \ldots ; x_{1}^{m-1}, x_{2}^{m-1}, \ldots ; \ldots ; x_{1}^{1}, x_{2}^{1}, \ldots\right) .
$$

As $\alpha \rightarrow \infty$, this determinantal process converges to the hard edge scaling limit in the strong coupling regime.

This is equivalent to the following statement:

- For $1 \leq r, s \leq m-1$ we have the kernel (3.1) (with $m \rightarrow m-1$ on the right-hand side).

- For $1 \leq r \leq m-1, s=m$ we have

$\lim _{\alpha \rightarrow \infty}\left(2^{\frac{1}{2}} \alpha y^{\frac{1}{2}} K_{\infty, m}^{\text {interpol }}\left(r, x ; m, \alpha^{2} y^{2} ; \alpha\right) \frac{e^{2 \alpha^{2} y^{2}}}{2^{\frac{1}{2}} \pi^{\frac{1}{2}}}\right)=K_{\infty, m}^{\text {Ginibre }}(r, x ; m-1, y)+\mathbf{1}_{r, m-1} \delta(x-y)$.

- For $r=m, 1 \leq s \leq m-1$ we have

$$
\lim _{\alpha \rightarrow \infty}\left(2^{\frac{1}{2}} \alpha x^{\frac{1}{2}} K_{\infty, m}^{\text {interpol }}\left(m, \alpha^{2} x^{2} ; s, y ; \alpha\right) \frac{2^{\frac{1}{2}} \pi^{\frac{1}{2}}}{e^{2 \alpha^{2} x^{2}}}\right)=K_{\infty, m}^{\text {Ginibre }}(m-1, x ; s, y) .
$$

- Finally, for $r=m, s=m$ we have

$$
\lim _{\alpha \rightarrow \infty}\left(2 \alpha^{2} x^{\frac{1}{2}} y^{\frac{1}{2}} K_{\infty, m}^{\text {interpol }}\left(m, \alpha^{2} x^{2} ; m, \alpha^{2} y^{2} ; \alpha\right) \frac{e^{2 \alpha^{2} y^{2}}}{e^{2 \alpha^{2} x^{2}}}\right)=K_{\infty, m}^{\text {Ginibre }}(m-1, x ; m-1, y) .
$$

In all these formulas the variables $x$ and $y$ take values in a compact subset of $\mathbb{R}_{>0}$.

In particular, Theorem 4.1 implies that the determinantal process on $\mathbb{R}_{>0}$ defined by the correlation kernel $K_{\infty, m}^{\text {interpol }}(m, x ; m, y ; \alpha)$ is an interpolating (one-level) determinantal point process. It interpolates between the Meijer $G$-kernel process for $m$ independent matrices, and the Meijer $G$-kernel process for $m-1$ independent matrices (with contact interaction from the identification of level $m$ with level $m-1$ ).

\section{An integration FORMUla FOR COUPLED MATRICES}

Below we derive an integration formula related to the investigation of singular variables of coupled matrices, see Lemma 5.1. The obtained formula will be applied to multi-matrix models, in order to derive the corresponding joint densities.

Lemma 5.1. Assume that $\nu \geq 0$ is an integer, and $b>0$. Let $G$ be a complex matrix of size $(n+\nu) \times l$, where $l \geq n \geq 1$, and $X$ be a complex matrix of size $l \times n$, with non-zero squared singular values $x_{1}, \ldots, x_{n}$. Denote by $y(G X)=\left\{y_{1}, \ldots, y_{n}\right\}$ the set of the squared singular values of matrix $Y=G X$. The complex flat Lebesgue measure for matrix $G$ is denoted by $d G$, i.e. $d G=\prod_{j=1}^{l} \prod_{k=1}^{n+\nu} d G_{j, k}^{R} d G_{j, k}^{I}, G_{j, k}=G_{j, k}^{R}+i G_{j, k}^{I}$ denotes the sum of the real and imaginary parts of the matrix entries $G_{j, k}$, Let $f($. be a continuous function on $\mathbb{R}_{>0}^{n}$ with compact support. In addition, we assume that 
$f(y)=f\left(y_{1}, \ldots, y_{n}\right)$ is symmetric with respect to permutations of $y_{1}, \ldots, y_{n}$. Finally, $V$ (the potential) is some scalar positive function, such that all the following three matrix integrals exist.

(A) We have for $l=n$

$$
\begin{aligned}
& \int f(y(G X)) e^{-\operatorname{Tr}\left[G^{*} G\right]+b \operatorname{Tr}\left[G X+(G X)^{*}\right]-\operatorname{Tr} V\left((G X)^{*}(G X)\right)} d G \\
& =\frac{c}{\Delta_{n}\left(\left\{x_{j}\right\}\right)} \\
& \times \int_{0}^{\infty} \ldots \int_{0}^{\infty} f\left(y_{1}, \ldots, y_{n}\right) \operatorname{det}\left[\frac{e^{-\frac{y_{j}}{x_{k}}-V\left(y_{j}\right)}}{x_{k}}\right]_{j, k=1}^{n} \operatorname{det}\left[y_{j}^{\frac{k-1}{2}} I_{k-1}\left(2 b y_{j}^{\frac{1}{2}}\right)\right]_{j, k=1}^{n} d y_{1} \ldots d y_{n},
\end{aligned}
$$

where we recall that the Vandermonde determinant was defined in (2.10). The constant $c$ does not depend on the set $\left\{x_{1}, \ldots, x_{n}\right\}$.

(B) Without the coupling term $(b=0)$ and for general $l \geq n$ we have

$$
\begin{aligned}
& \int f(y(G X)) e^{-\operatorname{Tr}\left[G^{*} G\right]-\operatorname{Tr} V\left((G X)^{*}(G X)\right)} d G \\
& =\frac{c^{\prime}}{\Delta_{n}\left(\left\{x_{i}\right\}\right)} \int_{0}^{\infty} \ldots \int_{0}^{\infty} f\left(y_{1}, \ldots, y_{n}\right) \operatorname{det}\left[\frac{y_{j}^{\nu} e^{-\frac{y_{j}}{x_{k}}-V\left(y_{j}\right)}}{x_{k}^{\nu+1}}\right]_{j, k=1}^{n} \Delta_{n}\left(\left\{y_{i}\right\}\right) d y_{1} \ldots d y_{n} .
\end{aligned}
$$

Here the constant $c^{\prime}$ does not depend on the set $\left\{x_{1}, \ldots, x_{n}\right\}$.

(C) Without matrix $X$ and for $l=n$ we have:

$$
\begin{aligned}
& \int f(y(G)) e^{-\operatorname{Tr}\left[G^{*} G\right]-\operatorname{Tr} V\left(G^{*} G\right)} d G \\
& =c^{\prime \prime} \int_{0}^{\infty} \ldots \int_{0}^{\infty} f\left(y_{1}, \ldots, y_{n}\right) \Delta_{n}^{2}\left(\left\{y_{i}\right\}\right) \prod_{j=1}^{n} y_{j}^{\nu} e^{-y_{j}-V\left(y_{j}\right)} d y_{1} \ldots d y_{n},
\end{aligned}
$$

where here $y(G)=\left\{y_{1}, \ldots, y_{n}\right\}$ is the set of squared singular values of $G$ instead.

Proof. Consider the following measure

$$
P(G, X) d G=e^{-\operatorname{Tr}\left[G^{*} G\right]+b \operatorname{Tr}\left[G X+(G X)^{*}\right]-\operatorname{Tr} V\left((G X)^{*}(G X)\right)} d G .
$$

Following the analysis of Fischman et al. [20] we set

$$
X=U\left(\begin{array}{c}
X_{0} \\
0
\end{array}\right)
$$


where $U$ is an $(n+\nu) \times(n+\nu)$ unitary matrix, 0 is a $\nu \times n$ matrix consisting of zeros only, and $X_{0}$ is an $n \times n$ complex matrix. We have

$$
\begin{aligned}
& P(G, X) d G \\
& =e^{-\operatorname{Tr}\left[G^{*} G\right]+b \operatorname{Tr}\left[G U\left(\begin{array}{c}
X_{0} \\
0
\end{array}\right)+\left(\begin{array}{cc}
X_{0}^{*} & 0
\end{array}\right) U^{*} G^{*}\right]-\operatorname{Tr} V\left(\left(\begin{array}{cc}
X_{0}^{*} & 0
\end{array}\right) U^{*} G^{*} G U\left(\begin{array}{c}
X_{0} \\
0
\end{array}\right)\right)} d G .
\end{aligned}
$$

Set $\widehat{G}=G U$, and note that $\widehat{G}$ is a matrix of size $n \times(n+\nu)$. Use the invariance of the Lebesgue measure under unitary transformations to write

$$
P(G, X) d G=e^{-\operatorname{Tr}\left[\widehat{G}^{*} \widehat{G}\right]+b \operatorname{Tr}\left[\widehat{G}\left(\begin{array}{c}
X_{0} \\
0
\end{array}\right)+\left(\begin{array}{ll}
X_{0}^{*} & 0
\end{array}\right) \widehat{G}^{*}\right]-\operatorname{Tr} V\left(\left(\begin{array}{cc}
X_{0}^{*} & 0
\end{array}\right) \widehat{G}^{*} \widehat{G}\left(\begin{array}{c}
X_{0} \\
0
\end{array}\right)\right)} d \widehat{G},
$$

where $d \widehat{G}=\prod_{j=1}^{n} \prod_{k=1}^{n+\nu} d \widehat{G}_{j, k}^{R} d \widehat{G}_{j, k}^{I}$, and where $\widehat{G}_{j, k}=\widehat{G}_{j, k}^{R}+i \widehat{G}_{j, k}^{I}$ denotes the sum of the real and imaginary parts of the matrix entries $\widehat{G}_{j, k}$. Write $\widehat{G}$ as $\widehat{G}=\left(G_{0}, G_{1}\right)$, where $G_{0}$ is a matrix of size $n \times n$, and where $G_{1}$ is a matrix of size $n \times \nu$. We obtain the following decomposition of the measure $P(G, X) d G$

$$
P(G, X) d G=\left(e^{-\operatorname{Tr}\left[G_{0}^{*} G_{0}\right]+b \operatorname{Tr}\left[G_{0} X_{0}+G_{0}^{*} X_{0}^{*}\right]-\operatorname{Tr} V\left(\left(G_{0} X_{0}\right)^{*}\left(G_{0} X_{0}\right)\right)} d G_{0}\right) e^{-\operatorname{Tr}\left[G_{1}^{*} G_{1}\right]} d G_{1} .
$$

We have put brackets here to emphasise that the integrals over $G_{0}$ and $G_{1}$ decouple, with the latter giving only an additional multiplicative constant. The important observation is that the matrices $X$ and $X_{0}$ have the same singular values, and that the matrices $G X$ and $G_{0} X_{0}$ have the same singular values. Set $Y=G_{0} X_{0}$. We have $d G_{0}=\operatorname{det}^{-n}\left[X_{0}^{*} X_{0}\right] d Y$, which gives

$$
\begin{aligned}
& P(G, X) d G \\
& =\left(e^{-\operatorname{Tr}\left[\left(Y X_{0}^{-1}\right)^{*} Y X_{0}^{-1}\right]+b \operatorname{Tr}\left[Y+Y^{*}\right]-\operatorname{Tr} V\left(Y^{*} Y\right)} \operatorname{det}^{-n}\left[X_{0}^{*} X_{0}\right] d Y\right) e^{-\operatorname{Tr}\left[G_{1}^{*} G_{1}\right]} d G_{1} .
\end{aligned}
$$

Here, we have used that $X_{0}$ is invertible, which is ensured by its non-zero squared singular values.

The the singular value decomposition of the matrix $Y$ can be written as $Y=\widetilde{U} \Sigma P$, where both $\widetilde{U}, P$ are unitary matrices of the same size $n \times n$, and where $\Sigma$ is an $n \times n$ diagonal matrix with a real matrix entries,

$$
\Sigma=\left(\begin{array}{cccc}
\sqrt{y_{1}} & 0 & \cdots & 0 \\
0 & \sqrt{y_{2}} & \cdots & 0 \\
\vdots & & & \\
0 & 0 & 0 & \sqrt{y_{n}}
\end{array}\right)
$$

and $y_{1}, \ldots, y_{n}$ are the squared singular values of $Y$. The Jacobian corresponding to this decomposition is

$$
d Y=c_{1} \Delta_{n}\left(\left\{y_{j}\right\}\right)^{2} d \widetilde{U} d P d y_{1} \ldots d y_{n}
$$


where $c_{1}$ is some constant. We thus obtain

$$
\begin{aligned}
P(G, X) d G= & c_{1} e^{-\operatorname{Tr}\left[P^{*} \Sigma^{2} P\left(X_{0}^{*} X_{0}\right)^{-1}\right]+b \operatorname{Tr}\left[\widetilde{U} \Sigma P+P^{*} \Sigma \widetilde{U}^{*}\right]-\operatorname{Tr} V\left(\Sigma^{2}\right)} \operatorname{det}^{-n}\left[X_{0}^{*} X_{0}\right] \Delta_{n}\left(\left\{y_{j}\right\}\right)^{2} d \widetilde{U} d V \\
& \times e^{-\operatorname{Tr}\left[G_{1}^{*} G_{1}\right]} d G_{1} .
\end{aligned}
$$

The next step is to use the invariance of the Haar measure under left shifts by the group elements, $\widetilde{U} \rightarrow \hat{U}=P \widetilde{U}$, and to rewrite the expression above as

$$
\begin{aligned}
P(G, X) d G= & c_{1} e^{-\operatorname{Tr}\left[P^{*} \Sigma^{2} P\left(X_{0}^{*} X_{0}\right)^{-1}\right]+b \operatorname{Tr}\left[\hat{U} \Sigma+\Sigma \hat{U}^{*}\right]-\operatorname{Tr} V\left(\Sigma^{2}\right)} \operatorname{det}^{-n}\left[X_{0}^{*} X_{0}\right] \Delta_{n}\left(\left\{y_{j}\right\}\right)^{2} d \hat{U} d P \\
& \times e^{-\operatorname{Tr}\left[G_{1}^{*} G_{1}\right]} d G_{1} .
\end{aligned}
$$

The integration over $P$ can be performed using the Harish-Chandra-Itzykson-Zuber integral formula [24, 27]

$$
\int_{U(n)} e^{-\operatorname{Tr}\left[P^{*} \Sigma^{2} P\left(X_{0}^{*} X_{0}\right)^{-1}\right]} d P=\text { const } \frac{\operatorname{det}\left[e^{-\frac{y_{j}}{x_{k}}}\right]_{j, k=1}^{n}}{\triangle\left(\left\{y_{j}\right\}\right) \Delta_{n}\left(\left\{x_{j}^{-1}\right\}\right)}
$$

where the constant depends only on $n$. The integration over $\hat{U}$ can be done exploiting the following integration formula [16] (sometimes called Leutwyler-Smilga formula [32])

$$
\int_{U(n)} e^{b \operatorname{Tr}\left[\Sigma\left(\hat{U}+\hat{U}^{*}\right)\right]} d \hat{U}=\mathrm{const} \frac{\operatorname{det}\left[y_{j}^{\frac{k-1}{2}} I_{k-1}\left(2 b y_{j}^{\frac{1}{2}}\right)\right]_{j, k=1}^{n}}{\Delta_{n}\left(\left\{y_{j}\right\}\right)}
$$

where $I_{\kappa}(x)$ denotes the modified Bessel function of the first kind. After integration, and after some simplifications, $\Delta_{n}\left(\left\{x_{j}^{-1}\right\}\right)=(-1)^{\frac{n(n-1)}{2}} \prod_{k=1}^{n} x_{k}^{-n+1} \Delta_{n}\left(\left\{x_{j}\right\}\right)$, we obtain formula (5.1) in the statement of the Lemma. Formula (5.2) can be obtained in the same way, and formula (5.3) is well known.

Proposition 5.2. Let $\nu, X, G$ be as in the statement of Lemma 5.1, and consider the probability measure

$$
P(G, X) d G=\frac{1}{Z_{n}} e^{-\operatorname{Tr}\left[G^{*} G\right]+b \operatorname{Tr}\left[G X+(G X)^{*}\right]} d G
$$

over rectangular complex matrices $G$. Here, $d G$ is the flat complex Lebesgue measure, and $Z_{n}$ is a normalising constant. Then, the density of the squared singular values $y_{1}, \ldots, y_{n}$ of $Y=G X$ is

$$
P\left(y_{1}, \ldots, y_{n}\right) d y_{1} \ldots d y_{n}=\frac{\prod_{l=1}^{n} x_{l}^{-1} e^{-b^{2} x_{l}}}{b^{\frac{n(n-1)}{2}}} \frac{\operatorname{det}\left[e^{-\frac{y_{j}}{x_{k}}}\right]_{j, k=1}^{n} \operatorname{det}\left[y_{j}^{\frac{k-1}{2}} I_{k-1}\left(2 b y_{j}\right)\right]_{j, k=1}^{n}}{n ! \Delta_{n}\left(\left\{x_{j}\right\}\right)} .
$$


Proof. By applying Lemma 5.1 (A) with $V=0$ the distribution follows. We only need to compute the following integral in order to determine the normalisation constant:

$$
I_{n}=\int_{0}^{\infty} \ldots \int_{0}^{\infty} \operatorname{det}\left[e^{-\frac{y_{j}}{x_{k}}}\right]_{j, k=1}^{n} \operatorname{det}\left[y_{j}^{\frac{k-1}{2}} I_{k-1}\left(2 b y_{j}^{\frac{1}{2}}\right)\right]_{j, k=1}^{n} d y_{1} \ldots d y_{n} .
$$

Applying the Andréief integral identity

$$
\int \cdots \int \operatorname{det}\left[\varphi_{i}\left(y_{j}\right)\right]_{i, j=1}^{n} \operatorname{det}\left[\psi_{i}\left(y_{j}\right)\right]_{i, j=1}^{n} d y_{1} \cdots d y_{n}=n ! \operatorname{det}\left[\int \varphi_{i}(y) \psi_{j}(y) d y\right]_{i, j=1}^{n},
$$

where the two sets of functions $\varphi_{i}$ and $\psi_{i}$ are assumed to be such that all integrals exist, we find that

$$
I_{n}=n ! \operatorname{det}\left[\int_{0}^{\infty} e^{-\frac{y}{x_{k}}} y^{\frac{j-1}{2}} I_{j-1}\left(2 b y^{\frac{1}{2}}\right) d y\right]_{j, k=1}^{n} .
$$

The integral inside the determinant can be computed explicitly. The result is [23, 6.631.4]

$$
\int_{0}^{\infty} e^{-\frac{y}{x_{k}}} y^{\frac{j-1}{2}} I_{j-1}\left(2 b y^{\frac{1}{2}}\right) d y=\left(x_{k}\right)^{j} b^{j-1} e^{b^{2} x_{k}}
$$

This gives

$$
I_{n}=b^{\frac{n(n-1)}{2}}\left(\prod_{k=1}^{n} x_{k} e^{b^{2} x_{k}}\right) \Delta_{n}\left(\left\{x_{j}\right\}\right) .
$$

The formula in the statement of Proposition 5.2 follows immediately.

Note that if $b=0$, then Proposition 5.2 reduces to the following known result, cf. Kuijlaars and Stivigny [30, Lemma 2.2] and references therein. Let $G$ be a complex Ginibre matrix of size $n \times(n+\nu)$, and let $X$ be a fixed complex matrix of size $(n+\nu) \times n$ with nonzero squared singular values $x(X)=\left(x_{1}, \ldots, x_{n}\right)$. Then the squared singular values $y_{1}, \ldots, y_{n}$ of matrix $Y=G X$ have a joint probability density proportional to

$$
\frac{\Delta_{n}\left(\left\{y_{j}\right\}\right)}{\Delta_{n}\left(\left\{x_{j}\right\}\right)} \operatorname{det}\left[\frac{e^{-\frac{y_{j}}{x_{k}}}}{x_{k}}\right]_{j, k=1}^{n} .
$$

\section{Proof of Theorem 2.1}

Set $G=G_{m}$, of size $n \times\left(n+\nu_{m-1}\right)$ (with $\left.\nu_{m}=0\right)$, and $X=G_{m-1} \cdots G_{1}$ of size $\left(n+\nu_{m-1}\right) \times n\left(\right.$ with $\left.\nu_{0}=0\right)$. Denote by $y(G X)$ the vector $y^{m}=\left(y_{1}^{m}, \ldots, y_{n}^{m}\right)$, which is the vector of the squared singular values of the matrix $Y_{m}=G X=G_{m} G_{m-1} \cdots G_{1}$. Note that $y^{m-1}=\left(y_{1}^{m-1}, \ldots, y_{n}^{m-1}\right)$ is the vector of the squared values of $X$. Let

$$
f: \underbrace{\left(\mathbb{R}_{>0}\right)^{n} \times \ldots \times\left(\mathbb{R}_{>0}\right)^{n}}_{m \text { times }} \longrightarrow \mathbb{C}
$$


be a continuous function with compact support, and denote by $\mathbb{E}[f]$ the expectation with respect to the probability measure defined by equation (2.1). Using this notation we can write

$$
\begin{aligned}
& \mathbb{E}\left[f\left(y^{m} ; \ldots ; y^{1}\right)\right] \\
& =\frac{1}{Z_{n}} \int\left(\int f\left(y(G X) ; y^{m-1} ; \ldots ; y^{1}\right) e^{-\operatorname{Tr}\left[G^{*} G\right]+b \operatorname{Tr}\left[G X+(G X)^{*}\right]-\operatorname{Tr} V_{m}\left((G X)^{*}(G X)\right)} d G\right) \\
& \times e^{-\sum_{l=1}^{m-1} \operatorname{Tr}\left[G_{l}^{*} G_{l}\right]-\sum_{l=1}^{m-1} \operatorname{Tr} V_{l}\left(\left(G_{l} \cdots G_{1}\right)^{*}\left(G_{l} \cdots G_{1}\right)\right)} \prod_{l=1}^{m-1} d G_{l} .
\end{aligned}
$$

The application of Lemma 5.1 (more explicitly, of equation (5.1)) to the integral over $G$ in the equation just written above gives

$$
\begin{aligned}
& \mathbb{E}\left[f\left(y^{m} ; \ldots ; y^{1}\right)\right] \\
& =\int f_{1}\left(y^{m-1} ; \ldots ; y^{1}\right) e^{-\sum_{l=1}^{m-1} \operatorname{Tr}\left[G_{l}^{*} G_{l}\right]-\sum_{l=1}^{m-1} \operatorname{Tr} V_{l}\left(\left(G_{l} \cdots G_{1}\right)^{*}\left(G_{l} \cdots G_{1}\right)\right)} \prod_{l=1}^{m-1} d G_{l},
\end{aligned}
$$

where

$$
\begin{aligned}
& f_{1}\left(y^{m-1} ; \ldots ; y^{1}\right) \\
& =\frac{c_{m}}{Z_{n}} \int_{\mathbb{R}_{>0}^{n}} f\left(y^{m} ; \ldots, y^{1}\right) \operatorname{det}\left[\frac{e^{-\frac{y_{j}^{m}}{y_{k}^{m-1}-V_{m}\left(y_{j}^{m}\right)}}}{y_{k}^{m-1}}\right]_{j, k=1}^{n} \frac{\operatorname{det}\left[\left(y_{j}^{m}\right)^{\frac{k-1}{2}} I_{k-1}\left(2 b\left(y_{j}^{m}\right)^{\frac{1}{2}}\right)\right]_{j, k=1}^{n}}{\Delta_{n}\left(\left\{y_{i}^{m-1}\right\}\right)} d y^{m} .
\end{aligned}
$$

Here, we denote by $d y^{m}=d y_{1}^{m} \cdots d y_{n}^{m}$ the integration measure over all squared singular values.

Next, let us apply Lemma 5.1, equation (5.2) to equation (6.1), with $G=G_{m-1}$ of size $\left(n+\nu_{m-1}\right) \times\left(n+\nu_{m-2}\right)$, and $X=G_{m-2} \cdots G_{1}$ of size $\left(n+\nu_{m-2}\right) \times n$, and integrate over $G_{m-1}$. The result can be written as

$$
\begin{aligned}
& \mathbb{E}\left[f\left(y^{m} ; \ldots ; y^{1}\right)\right] \\
& =\int f_{2}\left(y^{m-2} ; \ldots ; y^{1}\right) e^{-\sum_{l=1}^{m-2} \operatorname{Tr}\left[G_{l}^{*} G_{l}\right]-\sum_{l=1}^{m-2} \operatorname{Tr} V_{l}\left(\left(G_{l} \cdots G_{1}\right)^{*}\left(G_{l} \cdots G_{1}\right)\right)} \prod_{l=1}^{m-2} d G_{l},
\end{aligned}
$$


where

$$
\begin{aligned}
& f_{2}\left(y^{m-2} ; \ldots ; y^{1}\right) \\
& =\frac{c_{m} c_{m-1}}{Z_{n}} \int_{\mathbb{R}_{>0}^{n}} \int_{\mathbb{R}_{>0}^{n}} f\left(y^{m} ; \ldots, y^{1}\right) \operatorname{det}\left[\frac{e^{-\frac{y_{j}^{m}}{y_{k}^{m-1}}-V_{m}\left(y_{j}^{m}\right)}}{y_{k}^{m-1}}\right]_{j, k=1}^{n} \\
& \times \operatorname{det}\left[\frac{\left(y_{j}^{m-1}\right)^{\nu_{m}-1} e^{-\frac{y_{j}^{m-1}}{y_{k}^{m-2}-V_{m-1}\left(y_{j}^{m-1}\right)}}}{\left(y_{k}^{m-2}\right)^{\nu_{m-1}+1}}\right]_{j, k=1}^{n} \frac{\operatorname{det}\left[\left(y_{j}^{m}\right)^{\frac{k-1}{2}} I_{k-1}\left(2 b\left(y_{j}^{m}\right)^{\frac{1}{2}}\right)\right]_{j, k=1}^{n}}{\Delta_{n}\left(\left\{y_{i}^{m-2}\right\}\right)} d y^{m-1} d y^{m} .
\end{aligned}
$$

Repeating this procedure $m-3$ times, we get

$$
\mathbb{E}\left[f\left(y^{m} ; \ldots ; y^{1}\right)\right]=\int f_{m-1}\left(y^{1}\right) e^{-\operatorname{Tr}\left[G_{1}^{*} G_{1}\right]-\operatorname{Tr} V_{1}\left(G_{1}^{*} G_{1}\right)} d G_{1},
$$

where

$$
\begin{aligned}
& f_{m-1}\left(y^{1}\right) \\
& =\frac{\prod_{l=2}^{m} c_{l}}{Z_{n} \Delta_{n}\left(\left\{y_{i}^{1}\right\}\right)} \int_{\left(\mathbb{R}_{>0}^{n}\right)^{m-1}} f\left(y^{m} ; \ldots, y^{1}\right) \operatorname{det}\left[\left(y_{j}^{m}\right)^{\frac{k-1}{2}} I_{k-1}\left(2 b\left(y_{j}^{m}\right)^{\frac{1}{2}}\right)\right]_{j, k=1}^{n} \\
& \times \prod_{l=1}^{m-1} \operatorname{det}\left[\frac{\left(y_{j}^{l+1}\right)^{\nu_{l+1}}}{\left(y_{k}^{l}\right)^{\nu_{l+1}+1}} e^{-\frac{y_{j}^{l+1}}{y_{k}^{l}}-V_{l+1}\left(y_{j}^{l+1}\right)}\right]_{j, k=1}^{n} d y^{2} \ldots d y^{m} .
\end{aligned}
$$

The result of Theorem 2.1 follows by application of formula (5.3) to equation (6.3), and by taking into account equation (6.4). Eq. (2.3) is obtained by redistributing the factors with the potentials among the determinants, including the remaining Vandermonde determinant. Finally the normalisation constant $Z_{n, m}$ in (2.4) is obtained by an $m$-fold application of the Andréief formula (5.6).

\section{Proof of Proposition 2.5}

The integration of the density (2.20) of $y=\left(y^{m}, y^{m-1}, \ldots, y^{1}\right)$ over the sets of variables $y^{m-1}, \ldots, y^{1}$ gives Eq. (2.21) by applying the Andréief formula (5.6) $m-1$ times, with

$$
\begin{aligned}
\psi_{k}(y)= & \int_{0}^{\infty} \ldots \int_{0}^{\infty} t^{\nu_{1}+k-1}\left(\frac{t_{2}}{t_{1}}\right)^{\nu_{2}} \ldots\left(\frac{t_{m-1}}{t_{m-2}}\right)^{\nu_{m-1}}\left(\frac{y}{t_{m-1}}\right)^{\nu_{m}} \\
& \times e^{-t_{1}-\frac{t_{2}}{t_{1}}-\ldots-\frac{t_{m-1}}{t_{m-2}}-\frac{y}{t_{m-1}}-b^{2} t_{m-1}} \frac{d t_{1}}{t_{1}} \frac{d t_{2}}{t_{2}} \ldots \frac{d t_{m-1}}{t_{m-1}} .
\end{aligned}
$$


We note that the integration over $t_{1}, t_{2}, \ldots, t_{m-2}$ in the formula just written above results into the Meijer $G$-function (see e.g. [5]), namely

$$
\psi_{k}(y)=\int_{0}^{\infty} G_{0, m-1}^{m-1,0}\left(\begin{array}{cccc} 
& - & & \\
\nu_{1}+k-1 & \nu_{2} & \ldots & \nu_{m-1}
\end{array} \mid t_{m-1}\right) e^{-\frac{y}{t_{m-1}}-b^{2} t_{m-1}} \frac{d t_{m-1}}{t_{m-1}} .
$$

Using the contour integral representation for the Meijer $G$-function, cf. [35],

$$
\begin{aligned}
& G_{0, m-1}^{m-1,0}\left(\begin{array}{cccc} 
& - & & \\
\nu_{1}+k-1 & \nu_{2} & \ldots & \nu_{m-1}
\end{array} \mid t_{m-1}\right) \\
& =\frac{1}{2 \pi i} \int_{c-i \infty}^{c+i \infty} \Gamma\left(u+\nu_{1}+k-1\right) \Gamma\left(u+\nu_{2}\right) \ldots \Gamma\left(u+\nu_{m-1}\right)\left(t_{m-1}\right)^{-u} d u \text {, }
\end{aligned}
$$

with $c>0$, and taking into account that (2.16)

$$
\int_{0}^{\infty}\left(t_{m-1}\right)^{-u-1} e^{-\frac{y}{t_{m-1}}-b^{2} t_{m-1}} d t_{m-1}=2\left(\frac{y}{b^{2}}\right)^{-\frac{u}{2}} K_{u}(2 b \sqrt{y})
$$

we obtain equation (2.22). The interchange of integrals can be justified with Fubini's Theorem.

The normalisation constant as given in (2.23) can be obtained as follows. Applying the Andréief formula (5.6) to (2.21) once, we need to compute the determinant of the following integral

$$
a_{i, j}(b)=\int_{0}^{\infty} y^{\frac{j-1}{2}} I_{j-1}\left(2 b y^{\frac{1}{2}}\right) \psi_{i}(y) d y
$$

Using the representation of the function $\psi_{j}(y)$ from (7.1), we observe that we can use the integral (5.7) (with $x_{k}$ replaced by $t_{m-1}$ ) to obtain

$$
a_{i, j}(b)=b^{j-1} \int_{0}^{\infty} \ldots \int_{0}^{\infty} t^{\nu_{1}+i-1}\left(\frac{t_{2}}{t_{1}}\right)^{\nu_{2}} \ldots\left(\frac{t_{m-1}}{t_{m-2}}\right)^{\nu_{m-1}}\left(t_{m-1}\right)^{j} e^{-t_{1}-\frac{t_{2}}{t_{1}}-\ldots-\frac{t_{m-1}}{t_{m-2}}} \frac{d t_{1}}{t_{1}} \ldots \frac{d t_{m-1}}{t_{m-1}} .
$$

The powers in $b$ can be taken out of the determinant of $a_{i, j}(b)$, and the remaining integral is the same as in the normalisation of $m-1$ product of independent Ginibre matrices. For these the determinant has been computed in [5] and we thus obtain for

$$
\operatorname{det}\left[a_{i, j}(b)\right]_{i, j=1}^{n}=b^{\frac{n(n-1)}{2}} \prod_{j=1}^{n} \prod_{l=1}^{m-1} \Gamma\left(j+\nu_{l}\right) .
$$

Together with the $n$ ! from the Andréief formula (5.6) we obtain (2.23). 


\section{Measures given by Products of Determinants, Eynard-Mehta ThEOREM, AND PROOF OF THEOREM 2.7}

The aim of this section is to prove Theorem 2.7. Recall that Theorem 2.7 states that the product matrix process associated with probability measure (2.1), defined in Section 2.1, is a multi-level determinantal process living on $\{1, \ldots, m\} \times \mathbb{R}_{>0}$. Moreover, Theorem 2.7 gives a formula for the relevant correlation kernel.

The starting point of the proof of Theorem 2.4 is the fact that the density of the product matrix process under considerations is given by a product of determinants, see Theorem 2.1. This enables us to apply the Eynard-Mehta Theorem to the product matrix process.

Let us first recall the formulation of the Eynard-Mehta Theorem. Here we follow the elegant presentation of the Eynard-Mehta Theorem in Johansson [28]5.

Let $n, m \geq 1$ be two fixed natural numbers, and let $\mathfrak{X}_{0}, \mathfrak{X}_{m+1}$ be two given sets. Let $\mathfrak{X}$ be a complete separable metric space, and consider a probability measure on $\left(\mathfrak{X}^{n}\right)^{m}$ given by

$$
\begin{aligned}
p_{n, m}(\underline{x}) d \mu(\underline{x}) & =\frac{1}{Z_{N, m}} \operatorname{det}\left[\phi_{0,1}\left(x_{i}^{0}, x_{j}^{1}\right)\right]_{i, j=1}^{n} \operatorname{det}\left[\phi_{m, m+1}\left(x_{i}^{m}, x_{j}^{m+1}\right)\right]_{i, j=1}^{n} \\
& \times \prod_{r=1}^{m-1} \operatorname{det}\left[\phi_{r, r+1}\left(x_{i}^{r}, x_{j}^{r+1}\right)\right]_{i, j=1}^{n} d \mu(\underline{x}) .
\end{aligned}
$$

In the formula just written above $Z_{N, m}$ is the normalisation constant, the functions $\phi_{r, r+1}: \mathfrak{X} \times \mathfrak{X} \rightarrow \mathbb{C}, r=1, \ldots, m-1$ are given intermediate one-step transition functions, $\phi_{0,1}: \mathfrak{X}_{0} \times \mathfrak{X} \rightarrow \mathbb{C}$ is a given initial one-step transition function, and $\phi_{m, m+1}: \mathfrak{X} \times \mathfrak{X}_{m+1} \rightarrow$ $\mathbb{C}$ is a given final one-step transition function. Also,

$$
\underline{x}=\left(x^{1}, \ldots, x^{m}\right) \in\left(X^{n}\right)^{m} ; x^{r}=\left(x_{1}^{r}, \ldots, x_{n}^{r}\right), r=1, \ldots, m,
$$

the vectors

$$
x^{0}=\left(x_{1}^{0}, \ldots, x_{n}^{0}\right) \in \mathfrak{X}_{0}^{n}, x^{m+1}=\left(x_{1}^{m+1}, \ldots, x_{n}^{m+1}\right) \in \mathfrak{X}_{m+1}^{n},
$$

are fixed initial and final vectors, and

$$
d \mu(\underline{x})=\prod_{r=1}^{m} \prod_{j=1}^{n} d \mu\left(x_{j}^{r}\right) .
$$

Here, $\mu$ is a given Borel measure on $\mathfrak{X}$. Given two transition functions $\phi$ and $\psi$ set

$$
\phi * \psi(x, y)=\int_{\mathfrak{X}} \phi(x, t) \psi(t, y) d \mu(t) .
$$

\footnotetext{
${ }^{5}$ For other presentations of the Eynard-Mehta Theorem, and for different proofs we refer the reader to Borodin [15], Tracy and Widom [42.
} 
Proposition 8.1. Consider the probability measure defined by equation (8.1). The distribution of the vector $x^{m}=\left(x_{1}^{m}, \ldots, x_{n}^{m}\right)$ is given by

$$
\begin{aligned}
& p\left(x_{1}^{m}, \ldots, x_{n}^{m}\right) d \mu\left(x_{1}^{m}\right) \ldots d \mu\left(x_{n}^{m}\right) \\
& =\frac{1}{Z_{n, m}^{\prime}} \operatorname{det}\left[\phi_{0, m}\left(x_{i}^{0}, x_{j}^{m}\right)\right]_{i, j=1}^{n} \operatorname{det}\left[\phi_{m, m+1}\left(x_{i}^{m}, x_{j}^{m+1}\right)\right]_{i, j=1}^{n} d \mu\left(x_{1}^{m}\right) \ldots d \mu\left(x_{n}^{m}\right),
\end{aligned}
$$

where

$$
\phi_{0, m}(x, y)=\phi_{0,1} * \ldots * \phi_{m-1, m}(x, y),
$$

for $m>1$, and where the normalisation constant is given by

$$
Z_{n, m}^{\prime}=n ! \operatorname{det}\left[\phi_{0, m+1}\left(x_{i}^{0}, x_{j}^{m+1}\right)\right]_{i, j=1}^{n} .
$$

Proof. The density of $\left(x_{1}^{m}, \ldots, x_{n}^{m}\right)$ can be obtained by subsequent integration of the measure $p_{n, m}(\underline{x}) d \mu(\underline{x})$ over $x^{1}, \ldots, x^{m-1}$, and by application of the Andréief integral identity (5.6).

Let us define the following correlation functions for the process defined by probability measure (8.1):

$$
\varrho_{k_{1}, \ldots, k_{m}}\left(x_{1}^{1}, \ldots, x_{k_{1}}^{1} ; \ldots ; x_{1}^{m}, \ldots, x_{k_{m}}^{m}\right)=\prod_{j=1}^{m} \frac{n !}{\left(n-k_{j}\right) !} \int_{\mathfrak{X}} \cdots \int_{\mathfrak{X}} p_{n, m}(\underline{x}) \prod_{r=1}^{m} \prod_{j=k_{r}+1}^{n} d \mu\left(x_{j}^{r}\right) .
$$

The following statement determines these for the point process (8.1) and is often referred as the Eynard-Mehta Theorem [18].

Theorem 8.2. The probability measure $p_{n, m}(\underline{x}) d \mu(\underline{x})$ given by equation (8.1) defines a determinantal point process on $\{1, \ldots, m\} \times \mathfrak{X}$. The correlation kernel of this determinantal point process, $K_{n, m}(r, x ; s, y)$ (where $r, s \in\{1, \ldots, m\}$, and $\left.x, y \in \mathfrak{X}\right)$, is given by the formula

$$
K_{n, m}(r, x ; s, y)=-\phi_{r, s}(x, y)+\sum_{i, j=1}^{n} \phi_{r, m+1}\left(x, x_{i}^{m+1}\right)\left(A^{-1}\right)_{i, j} \phi_{0, s}\left(x_{j}^{0}, y\right) .
$$

The additional transition functions $\phi_{r, s}$ with $s \neq r+1$, and the matrix $A=\left(a_{i, j}\right)$, with $i, j=1, \ldots, n$, are defined as follows in terms of the one-step transition functions $\phi_{r, r+1}$, with $r=0,1, \ldots, m$, of point process (8.1):

$$
\phi_{r, s}(x, y)= \begin{cases}\left(\phi_{r, r+1} * \ldots * \phi_{s-1, s}\right)(x, y), & 0 \leq r<s \leq m+1, \\ 0, & r \geq s\end{cases}
$$

and

$$
a_{i, j}=\phi_{0, m+1}\left(x_{i}^{0}, x_{j}^{m+1}\right)
$$


The correlation functions defined in (8.2) can be written as determinants of block matrices, namely

$$
\begin{aligned}
& \varrho_{k_{1}, \ldots, k_{m}}\left(x_{1}^{1}, \ldots, x_{k_{1}}^{1} ; \ldots ; x_{1}^{m}, \ldots, x_{k_{m}}^{m}\right) \\
& =\operatorname{det}\left[\begin{array}{ccc}
\left(K_{n, m}\left(1, x_{i}^{1} ; 1, x_{j}^{1}\right)\right)_{i=1, \ldots, k_{1}}^{j=1, \ldots, k_{1}} & \ldots & \left(K_{n, m}\left(1, x_{i}^{1} ; m, x_{j}^{m}\right)\right)_{i=1, \ldots, k_{1}}^{j=1, \ldots, k_{m}} \\
\vdots & & \\
\left(K_{n, m}\left(m, x_{i}^{m} ; 1, x_{j}^{1}\right)\right)_{i=1, \ldots, k_{m}}^{j=1, \ldots, k_{1}} & \ldots & \left(K_{n, m}\left(m, x_{i}^{m} ; m, x_{j}^{m}\right)\right)_{i=1, \ldots, k_{m}}^{j=1, \ldots, k_{m}}
\end{array}\right] \text {, }
\end{aligned}
$$

where $1 \leq k_{1}, \ldots, k_{m} \leq n$, and for $1 \leq l, p \leq m$

$$
\left(K_{n, m}\left(l, x_{i}^{l} ; p, x_{j}^{p}\right)\right)_{i=1, \ldots, k_{l}}^{j=1, \ldots, k_{p}}=\left(\begin{array}{ccc}
K_{n, m}\left(l, x_{1}^{l} ; p, x_{1}^{p}\right) & \ldots & K_{n, m}\left(l, x_{1}^{l} ; p, x_{k_{p}}^{p}\right) \\
\vdots & & \\
K_{n, m}\left(l, x_{k_{l}}^{l} ; p, x_{1}^{p}\right) & \ldots & K_{n, m}\left(l, x_{k_{l}}^{l} ; p, x_{k_{p}}^{p}\right)
\end{array}\right) .
$$

Remarks. In what follows the functions

$$
\phi_{0, s}(i, y), \quad 2 \leq s \leq m,
$$

will be called initial transition functions, and the functions

$$
\phi_{r, m+1}(x, j), \quad 1 \leq r \leq m-1,
$$

will be called final transition functions. In addition, the functions of the form

$$
\phi_{r, s}(x, y), \quad 1 \leq r \leq m-2, \quad r+2 \leq s \leq m,
$$

will be called intermediate transition functions. Finally, the function

$$
\phi_{0, m+1}(i, j)
$$

will be called the total transition function.

In order to prove Theorem 2.7 we need to rewrite the density of the product matrix process obtained in Theorem 2.1 as in the formulation of the Eynard-Mehta Theorem, see equation (8.1), and to obtain explicit expressions for the relevant transition functions. This is done below.

- One-step transition functions. Recall that $\nu_{0}=\nu_{m}=0$. In our situation $\mathfrak{X}_{0}=$ $\{1, \ldots, n\}, \mathfrak{X}_{m+1}=\{1, \ldots, n\}, \mathfrak{X}=\mathbb{R}_{>0}$, and $d \mu$ is the Lebesgue measure on $\mathbb{R}_{>0}$. The initial given one-step transition function is defined by

$$
\phi_{0,1}:\{1, \ldots, n\} \times \mathbb{R}_{>0} \rightarrow \mathbb{R}_{>0} ; \quad \phi_{0,1}(i, x)=x^{\nu_{1}+i-1} e^{-x} .
$$

The final given one-step transition function is defined by

$$
\phi_{m, m+1}: \mathbb{R}_{>0} \times\{1, \ldots, n\} \rightarrow \mathbb{R}_{>0} ; \quad \phi_{m, m+1}(x, k)=x^{\frac{k-1}{2}} I_{k-1}\left(2 b x^{\frac{1}{2}}\right) e^{-V_{m}(x)} .
$$

In addition, the intermediate given one-step transition functions

$$
\phi_{r, r+1}: \mathbb{R}_{>0} \times \mathbb{R}_{>0} \rightarrow \mathbb{R}_{>0}, \quad r=1, \ldots, m-1,
$$


are defined by

$$
\phi_{r, r+1}(x, y)=\left(\frac{y}{x}\right)^{\nu_{r+1}} \frac{e^{-\frac{y}{x}-V_{r}(x)}}{x}, \quad r=1, \ldots, m-1 .
$$

- Initial transition functions. The initial transition functions, $\phi_{0, s}(i, y)$, with $2 \leq$ $s \leq m$, can be written as

$$
\begin{aligned}
\phi_{0, s}(i, y) & =\phi_{0,1} * \phi_{1,2} * \ldots * \phi_{s-2, s-1} * \phi_{s-1, s}(i, y) \\
& =\int_{0}^{\infty} \ldots \int_{0}^{\infty} \phi_{0,1}\left(i, t_{1}\right) \phi_{1,2}\left(t_{1}, t_{2}\right) \ldots \phi_{s-2, s-1}\left(t_{s-2}, t_{s-1}\right) \phi_{s-1, s}\left(t_{s-1}, y\right) d t_{1} \ldots d t_{s-1},
\end{aligned}
$$

spelling out the convolution in the last line. Inserting the corresponding expressions for the one-step transition functions (8.7) and (8.9) we obtain

$$
\begin{aligned}
\phi_{0, s}(i, y)= & \int_{0}^{\infty} \ldots \int_{0}^{\infty} t_{1}^{\nu_{1}+i-1}\left(\frac{t_{2}}{t_{1}}\right)^{\nu_{2}} \ldots\left(\frac{t_{s-1}}{t_{s-2}}\right)^{\nu_{s-1}}\left(\frac{y}{t_{s-1}}\right)^{\nu_{s}} \\
& \times e^{-t_{1}-\frac{t_{2}}{t_{1}}-\ldots-\frac{t_{s-1}}{t_{s-2}}-\frac{y}{t_{s-1}}-V_{1}\left(t_{1}\right)-\ldots-V_{s-1}\left(t_{s-1}\right)} \frac{d t_{1}}{t_{1}} \ldots \frac{d t_{s-1}}{t_{s-1}}
\end{aligned}
$$

where $2 \leq s \leq m$ (for $s=1$ see (8.7)) .

- Final transition functions. The final transition functions $\phi_{r, m+1}(x, j)$, with $1 \leq r \leq m-1$, can be written in terms of the one-step transition functions as

$$
\begin{aligned}
& \phi_{r, m+1}(x, j) \\
& =\phi_{r, r+1} * \phi_{r+1, r+2} * \ldots * \phi_{m-1, m} * \phi_{m, m+1}(x, j) \\
& =\int_{0}^{\infty} \ldots \int_{0}^{\infty} \phi_{r, r+1}\left(x, t_{r+1}\right) \phi_{r+1, r+2}\left(t_{r+1}, t_{r+2}\right) \ldots \phi_{m-1, m}\left(t_{m-1}, t_{m}\right) \phi_{m, m+1}\left(t_{m}, j\right) d t_{r+1} \ldots d t_{m} .
\end{aligned}
$$

Inserting the explicit formulae for the one-step transition functions (8.9) and (8.8) we find

$$
\begin{aligned}
\phi_{r, m+1}(x, j)= & \frac{e^{-V_{r}(x)}}{x} \int_{0}^{\infty} \ldots \int_{0}^{\infty}\left(\frac{t_{r+1}}{x}\right)^{\nu_{r+1}}\left(\frac{t_{r+2}}{t_{r+1}}\right)^{\nu_{r+2}} \ldots\left(\frac{t_{m}}{t_{m-1}}\right)^{\nu_{m}}\left(t_{m}\right)^{\frac{j-1}{2}} I_{j-1}\left(2 b\left(t_{m}\right)^{\frac{1}{2}}\right) \\
& \times e^{-\frac{t_{r+1}}{x}-\frac{t_{r+2}}{t_{r+1}}-\ldots-\frac{t_{m}}{t_{m-1}}-V_{r+1}\left(t_{r+1}\right) \ldots-V_{m}\left(t_{m}\right)} \frac{d t_{r+1}}{t_{r+1}} \ldots \frac{d t_{m-1}}{t_{m-1}} d t_{m},
\end{aligned}
$$

where $1 \leq r \leq m-1($ for $r=m$ see $(\underline{8.8}))$. 
- Intermediate transition functions. Recall that the intermediate transition functions $\phi_{r, s}(x, y), 1 \leq r \leq m-2, r+2 \leq s \leq m$ are defined by

$$
\begin{aligned}
\phi_{r, s}(x, y) & =\phi_{r, r+1} * \phi_{r+1, r+2} * \ldots * \phi_{s-1, s}(x, y) \\
& =\int_{0}^{\infty} \ldots \int_{0}^{\infty} \phi_{r, r+1}\left(x, t_{r+1}\right) \phi_{r+1, r+2}\left(t_{r+1}, t_{r+2}\right) \ldots \phi_{s-1, s}\left(t_{s-1}, y\right) d t_{r+1} \ldots d t_{s-1},
\end{aligned}
$$

where $1 \leq r \leq m-2, r+2 \leq s \leq m$. Using the explicit formulae for the intermediate one-step transition functions $(\underline{8.9})$ we get

$$
\begin{aligned}
\phi_{r, s}(x, y)= & \frac{e^{-V_{r}(x)}}{x} \int_{0}^{\infty} \ldots \int_{0}^{\infty}\left(\frac{t_{r+1}}{x}\right)^{\nu_{r+1}}\left(\frac{t_{r+2}}{t_{r+1}}\right)^{\nu_{r+2}} \ldots\left(\frac{y}{t_{s-1}}\right)^{\nu_{s}} \\
& \times e^{-\frac{t_{r+1}}{x}-\frac{t_{r+2}}{t_{r+1}}-\ldots-\frac{t_{s-1}}{t_{s-2}}-\frac{y}{t_{s-1}}-V_{r+1}\left(t_{r+1}\right) \ldots-V_{s-1}\left(t_{s-1}\right)} \frac{d t_{r+1}}{t_{r+1}} \ldots \frac{d t_{s-1}}{t_{s-1}}
\end{aligned}
$$

where $1 \leq r \leq m-2, r+2 \leq s \leq m$ (for $s=r+1$ see (8.9)).

- Total transition function. The total transition function $\phi_{0, m+1}(i, j)=a_{i, j}$ that constitutes matrix $A$ can be written as the convolution of the one-step initial transition function $\phi_{0,1}$ from (8.7) and the final transition function $\phi_{1, m+1}$ from (8.13) for $r=1$ :

$$
\phi_{0, m+1}(i, j)=\phi_{0,1} * \phi_{1, m+1}(i, j)=\int_{0}^{\infty} \phi_{0,1}\left(i, t_{1}\right) \phi_{1, m+1}\left(t_{1}, j\right) d t_{1} .
$$

This gives

$$
\begin{aligned}
\phi_{0, m+1}(i, j) & =\int_{0}^{\infty} \ldots \int_{0}^{\infty} t_{1}^{\nu_{1}+i-1}\left(\frac{t_{2}}{t_{1}}\right)^{\nu_{2}} \ldots\left(\frac{t_{m}}{t_{m-1}}\right)^{\nu_{m}}\left(t_{m}\right)^{\frac{j-1}{2}} I_{j-1}\left(2 b\left(t_{m}\right)^{\frac{1}{2}}\right) \\
& \times e^{-t_{1}-\frac{t_{2}}{t_{1}}-\ldots-\frac{t_{m}}{t_{m-1}}-V_{1}\left(t_{1}\right)-\ldots-V_{m}\left(t_{m}\right)} \frac{d t_{1}}{t_{1}} \ldots \frac{d t_{m-1}}{t_{m-1}} d t_{m} .
\end{aligned}
$$

Once all the transition functions are written explicitly, Theorem 2.7 follows immediately from Theorem 8.2 .

\section{Double CONTOUR INTEGRAL REPRESENTATION FOR THE CORRELATION KERNEL}

In this section we consider the $m$-matrix model defined by probability measure (2.19). The correlation kernel of the product matrix process associated with this model is denoted by $K_{n, m}(r, x ; s, y ; b)$. Our aim is to derive a double contour integral representation for $K_{n, m}(r, x ; s, y ; b)$, and to prove Theorem 2.8. Note that the multi-matrix model defined by probability measure (2.1) turns into that defined by probability measure (2.19) if

$$
V_{1}(t)=\ldots=V_{m-2}(t)=0, \quad V_{m-1}(t)=b^{2} t, \quad V_{m}(t)=0 .
$$


Therefore, in order to derive a contour integral representation for the correlation kernel $K_{n, m}(r, x ; s, y ; b)$ (2.29) we can exploit the formulae obtained in Theorem 2.7 with the potential functions specified by (9.1).

Proposition 9.1. For the specific case $V_{1}(t)=\ldots=V_{m-2}(t)=0, V_{m-1}(t)=b^{2} t$, $V_{m}(t)=0$ the correlation kernel $K_{n, m}(r, x ; s, y ; b)$ can be written as

$$
K_{n, m}(r, x ; s, y ; b)=-\phi_{r, s}(x, y ; b)+\sum_{i, j=1}^{n} \phi_{r, m+1}(x, i)\left(A^{-1}\right)_{i, j} \phi_{0, s}(j, y) .
$$

Here, the three sets of functions are obtained as follows.

(i) The functions $\phi_{r, s}(x, y ; b)$ are given by

$$
\begin{aligned}
& \phi_{r, s}(x, y ; b)
\end{aligned}
$$

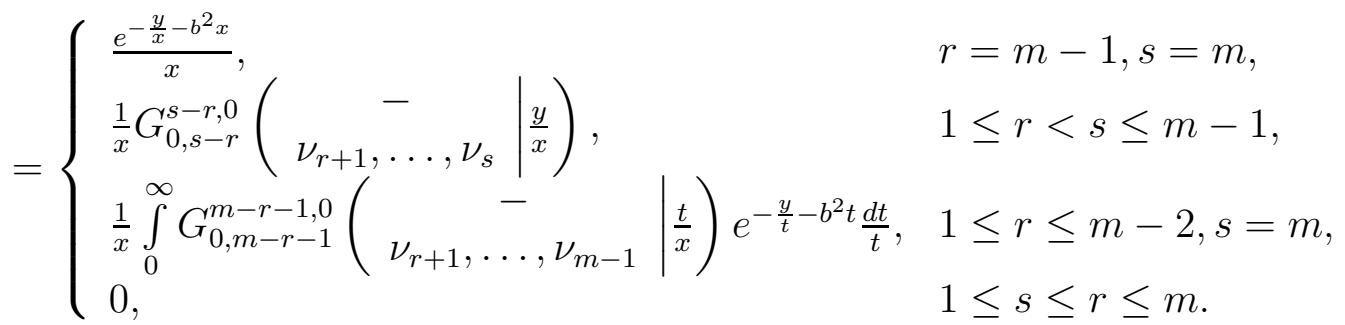

(ii) The functions $\phi_{r, m+1}(x, i)$ are given by

$$
\phi_{r, m+1}(x, j)= \begin{cases}b^{j-1} x^{j-1} \Gamma\left(j+\nu_{r+1}\right) \ldots \Gamma\left(j+\nu_{m-1}\right), & r \in\{1, \ldots, m-2\}, \\ b^{j-1} x^{j-1}, & r=m-1, \\ x^{\frac{j-1}{2}} I_{j-1}\left(2 b x^{\frac{1}{2}}\right), & r=m .\end{cases}
$$

(iii) The functions $\phi_{0, s}(i, y)$ are given by

$$
\phi_{0, s}(i, y)= \begin{cases}G_{0, s}^{s, 0}\left(\begin{array}{c}
- \\
\nu_{1}+i-1, \nu_{2}, \ldots, \nu_{s}
\end{array} \mid y\right) & s \in\{1, \ldots, m-1\} \\
\int_{0}^{\infty} G_{0, m-1}^{m-1,0}\left(\begin{array}{c}
\nu_{1}+i-1, \nu_{2}, \ldots, \nu_{m-1}
\end{array} \mid t\right) e^{-\frac{y}{t}-b^{2} t \frac{d t}{t}} & s=m .\end{cases}
$$

Finally, for the matrix $A=\left(a_{i, j}\right)_{i, j=1}^{n}$, we have

$$
a_{i, j}=\phi_{0, m+1}(i, j)=b^{j-1} \Gamma\left(i+j-1+\nu_{1}\right) \Gamma\left(j+\nu_{2}\right) \ldots \Gamma\left(j+\nu_{m-1}\right) .
$$

Proof. The formulae for the transition functions stated in Proposition 9.1 can be obtained by straightforward calculations starting from the formulae obtained in Theorem 2.7. In the calculations we have exploited the following integral representation of the Meijer 
$G$-function, cf. [5],

$$
\begin{aligned}
& G_{0, m}^{m, 0}\left(\begin{array}{c|c}
- & x_{m} \\
b_{1}, \ldots, b_{m} & x_{0}
\end{array}\right. \\
& =\int_{0}^{\infty} \ldots \int_{0}^{\infty}\left(\frac{x_{1}}{x_{0}}\right)^{b_{1}}\left(\frac{x_{2}}{x_{1}}\right)^{b_{2}} \ldots\left(\frac{x_{m}}{x_{m-1}}\right)^{b_{m}} e^{-\frac{x_{1}}{x_{0}}-\frac{x_{2}}{x_{1}}-\ldots-\frac{x_{m}}{x_{m-1}}} \frac{d x_{1}}{x_{1}} \ldots \frac{d x_{m-1}}{x_{m-1}},
\end{aligned}
$$

the fact that the Mellin transform of a Meijer $G$-function is given by

$$
\int_{0}^{\infty} t^{u-1} G_{p, q}^{m, n}\left(\begin{array}{c}
a_{1}, \ldots, a_{p} \\
b_{1}, \ldots, b_{q}
\end{array} \mid t z\right) d t=z^{-u} \frac{\prod_{i=1}^{m} \Gamma\left(b_{i}+u\right) \prod_{i=1}^{n} \Gamma\left(1-a_{i}-u\right)}{\prod_{i=n+1}^{p} \Gamma\left(a_{i}+u\right) \prod_{i=m+1}^{q} \Gamma\left(1-b_{i}-u\right)},
$$

see Luke [35], and the integral (5.7) involving the modified Bessel functions of the first kind

In Proposition 9.1 we have found the matrix entries of $A$ explicitly. This enables us to derive a formula for the inverse of $A$.

Proposition 9.2. Let $C=\left(c_{j, k}\right)_{j, k=1}^{n}$ be the inverse of $A=\left(a_{i, j}\right)_{i, j=1}^{n}$, where $a_{i, j}$ is given by equation (9.4). Thus the matrix elements $\left(c_{j, k}\right)_{j, k=1}^{n}$ are defined by the relation

$$
\sum_{j=1}^{n} a_{i, j} c_{j, k}=\delta_{i, k}, \quad i, k \in\{1, \ldots, n\}
$$

We have 6

$$
\begin{aligned}
c_{j, k}= & \frac{1}{b^{j-1} \Gamma\left(j+\nu_{2}\right) \ldots \Gamma\left(j+\nu_{m-1}\right)} \\
& \times \sum_{p=0}^{n-1} \frac{\Gamma\left(\nu_{1}+p+1\right)}{\Gamma\left(\nu_{1}+j\right) \Gamma\left(\nu_{1}+k\right) p !} \frac{(-p)_{j-1}(-p)_{k-1}}{\left(\nu_{1}+1\right)_{j-1}\left(\nu_{1}+1\right)_{k-1}} \frac{1}{(j-1) !(k-1) !} .
\end{aligned}
$$

Proof. Clearly, the matrix elements $\left(c_{j, k}\right)_{j, k=1}^{n}$ can be written as

$$
c_{j, k}=\frac{C_{j, k}}{b^{j-1} \Gamma\left(j+\nu_{2}\right) \ldots \Gamma\left(j+\nu_{m-1}\right)},
$$

where $C_{j, k}$ is defined by

$$
\sum_{j=1}^{n} \Gamma\left(i+j-1+\nu_{1}\right) C_{j, k}=\delta_{i, k}, \quad i, k \in\{1, \ldots, n\} .
$$

Next we use the fact that the inverse $\left(\alpha_{k, l}\right)_{k, l=0}^{N-1}$ of the Hankel matrix

$$
\left(h_{k+l}\right)_{k, l=0}^{N-1}, \quad h_{k}=\Gamma(k+\nu+1),
$$

\footnotetext{
${ }^{6}$ Recall that the Pochhammer symbol, $(a)_{l}$, is defined by $(a)_{l}=a(a+1) \ldots(a+l-1)$.
} 
is given by

$$
\alpha_{k, l}=\sum_{p=0}^{N-1} \frac{\Gamma(\nu+p+1)(-p)_{k}(-p)_{l}}{p ! \Gamma\left(\nu_{1}+k+1\right) \Gamma\left(\nu_{1}+l+1\right) k ! l !},
$$

see, for example, Akemann and Strahov [7, Section 5]. Equation (9.7) follows.

The fact that the inverse of the matrix $A$ can be written explicitly allows us to spell out the correlation kernel $K_{n, m}(r, x ; s, y ; b)$ of Proposition 9.1. We start from equation (9.2), use Proposition 9.2, and obtain the formula

$$
K_{n, m}(r, x ; s, y ; b)=-\phi_{r, s}(x, y ; b)+\sum_{p=0}^{n-1} \frac{\Gamma\left(\nu_{1}+p+1\right)}{\Gamma^{2}\left(\nu_{1}+1\right) p !} P_{r, p}(x) Q_{s, p}(y),
$$

where the transition functions $\phi_{r, s}(x, y)$ are defined in Proposition 9.1 (i),

$$
P_{r, p}(x)=\sum_{i=0}^{p} \phi_{r, m+1}(x, i+1) \frac{(-p)_{i}}{\Gamma\left(i+\nu_{2}+1\right) \ldots \Gamma\left(i+\nu_{m-1}+1\right)\left(\nu_{1}+1\right)_{i} i ! b^{i}}
$$

and

$$
Q_{s, p}(y)=\sum_{j=0}^{p} \phi_{0, s}(j+1, y) \frac{(-p)_{j}}{\left(\nu_{1}+1\right)_{j} j !} .
$$

Note that the Pochhammer symbol truncates both sums in $P_{r, p}(x)$ and $Q_{s, p}(y)$ that initially ran up to $n-1$. Proposition 9.1 gives the transition functions $\phi_{r, m+1}(x, i+1)$ and $\phi_{0, s}(j+1, y)$ explicitly. This enables us to obtain different, useful formulae for the functions $P_{r, p}(x)$ and $Q_{s, p}(y)$, involved in expression (9.8) for the correlation kernel $K_{n, m}(r, x ; s, y ; b)$.

- The functions $P_{r, p}(x)$. Let us derive a contour integration formula for the functions $P_{r, p}(x)$. If $r \in\{1, \ldots, m-1\}$, we have

$$
\begin{aligned}
P_{r, p}(x) & =\Gamma\left(\nu_{1}+1\right) \sum_{i=0}^{p} \frac{x^{i}(-p)_{i}}{i ! \Gamma\left(\nu_{1}+i+1\right) \ldots \Gamma\left(\nu_{r}+i+1\right)} \\
& =(-1)^{p} \Gamma\left(\nu_{1}+1\right) p ! \sum_{i=0}^{p} \frac{(-1)^{p-i} x^{i}}{i !(p-i) ! \Gamma\left(\nu_{1}+i+1\right) \ldots \Gamma\left(\nu_{r}+i+1\right)} .
\end{aligned}
$$

For $r=m$, the formula for the function $P_{m, p}(x)$ is

$$
\begin{aligned}
P_{m, p}(x) & =\Gamma\left(\nu_{1}+1\right) \sum_{i=0}^{p} \frac{x^{\frac{i}{2}}(-p)_{i}}{\Gamma\left(\nu_{1}+i+1\right) \ldots \Gamma\left(\nu_{m-1}+i+1\right)} \frac{I_{i}\left(2 b x^{\frac{1}{2}}\right)}{b^{i} i !} \\
& =(-1)^{p} \Gamma\left(\nu_{1}+1\right) p ! \sum_{i=0}^{p} \frac{(-1)^{p-i} x^{\frac{i}{2}}}{(p-i) ! \Gamma\left(\nu_{1}+i+1\right) \ldots \Gamma\left(\nu_{m-1}+i+1\right)} \frac{I_{i}\left(2 b x^{\frac{1}{2}}\right)}{b^{i} i !} .
\end{aligned}
$$


Using the expressions for the functions $P_{r, p}(x)$ just written above together with the Residue Theorem we immediately obtain a contour integral representation for these functions. Namely, for $r \in\{1, \ldots, m-1\}$ we find

$$
P_{r, p}(x)=\frac{(-1)^{p} \Gamma\left(\nu_{1}+1\right) p !}{2 \pi i} \oint_{\Sigma_{p}} \frac{\Gamma(t-p)}{\prod_{j=0}^{r} \Gamma\left(t+\nu_{j}+1\right)} x^{t} d t
$$

and for $r=m$ we find

$$
P_{m, p}(x)=\frac{(-1)^{p} \Gamma\left(\nu_{1}+1\right) p !}{2 \pi i} \oint_{\Sigma_{p}} \frac{\Gamma(t-p) x^{\frac{t}{2}}}{\prod_{j=0}^{m-1} \Gamma\left(t+\nu_{j}+1\right)} \frac{I_{t}\left(2 b x^{\frac{1}{2}}\right)}{b^{t}} d t .
$$

In the formulae just written above $\Sigma_{p}$ denotes a closed contour encircling $0,1, \ldots, p$ in positive direction.

- The functions $Q_{s, p}(y)$. For $s \in\{1, \ldots, m-1\}$ the formula for the functions $Q_{s, p}(y)$ reads

$$
Q_{s, p}(y)=\sum_{j=0}^{p} \frac{(-p)_{j} G_{0, s}^{s, 0}\left(\begin{array}{c}
- \\
\nu_{1}+j, \nu_{2} \ldots, \nu_{s}
\end{array} \mid y\right)}{\left(\nu_{1}+1\right)_{j} j !} .
$$

The contour integral representation of the Meijer $G$-function above is

$$
G_{0, s}^{s, 0}\left(\begin{array}{c}
- \\
\nu_{1}+j, \nu_{2} \ldots, \nu_{s}
\end{array} \mid y\right)=\frac{1}{2 \pi i} \int_{c-i \infty}^{c+i \infty} \Gamma\left(u+\nu_{1}+j\right) \Gamma\left(u+\nu_{2}\right) \ldots \Gamma\left(u+\nu_{s}\right) y^{-u} d u,
$$

for $c>0$ and $\nu_{j} \geq 0 \forall j$, leaving the poles of the Gamma-functions to the left of the contour. Therefore, we can write

$$
Q_{s, p}(y)=\frac{1}{2 \pi i} \int_{c-i \infty}^{c+i \infty} \Gamma\left(u+\nu_{1}\right) \ldots \Gamma\left(u+\nu_{s}\right)\left(\sum_{j=0}^{p} \frac{(-p)_{j}\left(u+\nu_{1}\right)_{j}}{\left(1+\nu_{1}\right)_{j} j !}\right) y^{-u} d u .
$$

The sum inside the integral above can be written as Gauss' hypergeometric function,

$$
\sum_{j=0}^{p} \frac{(-p)_{j}\left(u+\nu_{1}\right)_{j}}{\left(1+\nu_{1}\right)_{j}} \frac{1}{j !}={ }_{2} F_{1}\left(-p, u+\nu_{1} ; 1+\nu_{1} ; 1\right) \text {. }
$$

By the Chu-Vandermonde formula for the Gauss hypergeometric functions (see, for example, Ismail [26, Section 1.4])

$$
{ }_{2} F_{1}\left(-p, u+\nu_{1} ; 1+\nu_{1} ; 1\right)=\frac{(1-u)_{p}}{\left(1+\nu_{1}\right)_{p}}=(-1)^{p} \frac{\Gamma(u)}{\Gamma(u-p)} \frac{1}{\left(1+\nu_{1}\right)_{p}} .
$$


So we find

$$
Q_{s, p}(y)=\frac{(-1)^{p} \Gamma\left(1+\nu_{1}\right)}{2 \pi i \Gamma\left(1+\nu_{1}+p\right)} \int_{c-i \infty}^{c+i \infty} \frac{\prod_{j=0}^{s} \Gamma\left(u+\nu_{j}\right)}{\Gamma(u-p)} y^{-u} d u
$$

where $s \in\{1, \ldots, m-1\}$.

To obtain an integral representation for the function $Q_{m, p}(y)$ we proceed as follows. Recalling the definition (2.16)

$$
\int_{0}^{+\infty} e^{-\frac{\beta}{t}-\gamma t} t^{\nu-1} d t=2\left(\frac{\beta}{\gamma}\right)^{\frac{\nu}{2}} K_{\nu}(2 \sqrt{\beta \gamma}), \quad \beta>0, \gamma>0
$$

together with the contour integral representation for the corresponding Meijer $G$-function equation (9.16), applying Fubini's Theorem we obtain

$$
\phi_{0, m}(j+1, y)=\frac{1}{2 \pi i} \int_{c-i \infty}^{c+i \infty} \Gamma\left(u+\nu_{1}+j\right) \Gamma\left(u+\nu_{2}\right) \ldots \Gamma\left(u+\nu_{m-1}\right) 2 b^{u} y^{\frac{u}{2}} K_{u}\left(2 b y^{\frac{1}{2}}\right) y^{-u} d u .
$$

Inserting this into the formula for $Q_{m, p}(y)$, equation (9.10), gives

$$
Q_{m, p}(y)=\frac{1}{2 \pi i} \int_{c-i \infty}^{c+i \infty} \prod_{j=1}^{m-1} \Gamma\left(u+\nu_{j}\right)_{2} F_{1}\left(-p, u+\nu_{1} ; 1+\nu_{1} ; 1\right) 2 b^{u} y^{-\frac{u}{2}} K_{u}\left(2 b y^{\frac{1}{2}}\right) d u .
$$

By the same arguments as above, this formula can be rewritten as

$$
Q_{m, p}(y)=\frac{(-1)^{p} \Gamma\left(1+\nu_{1}\right)}{2 \pi i \Gamma\left(1+\nu_{1}+p\right)} \int_{c-i \infty}^{c+i \infty} \frac{\prod_{j=0}^{m} \Gamma\left(u+\nu_{j}\right)}{\Gamma(u-p)} \frac{2 b^{u} y^{-\frac{u}{2}} K_{u}\left(2 b y^{\frac{1}{2}}\right)}{\Gamma(u)} d u
$$

Now we are ready to prove Theorem 2.8. In order to derive the formula for the correlation kernel stated in the Theorem 2.8 it is enough to represent the sum

$$
S_{n, m}(r, x ; s, y ; b)=\sum_{p=0}^{n-1} \frac{\Gamma\left(\nu_{1}+p+1\right)}{\Gamma^{2}\left(\nu_{1}+1\right) p !} P_{r, p}(x) Q_{s, p}(y)
$$

as a double contour integral. For this purpose use the contour integral representations for the functions $P_{r, p}(x)$ and $Q_{s, p}(y)$ obtained above, equations (9.13), (9.14), (9.19), and (9.23), and the formula derived in [31, Eq.5.3]:

$$
\sum_{p=0}^{n-1} \frac{\Gamma(t-p)}{\Gamma(u-p)}=\frac{1}{u-t-1}\left[\frac{\Gamma(t-n+1)}{\Gamma(u-n)}-\frac{\Gamma(t+1)}{\Gamma(u)}\right] .
$$


Then note that by the Residue Theorem the second term in the right hand side of equation (9.24) does not contribute to $S_{n, m}(r, x ; s, y ; b)$. Here, we choose $c=\frac{1}{2}$, and due to the shift in argument of the function $q_{s}(u, y ; b)$ in equation (2.41) this leads to the contour as stated in Theorem 2.8.

Remarks. We would like to point out that for the special case $m=2$ and $r=s=2$ the kernel $K_{n, m}(r, x ; s, y ; b)$ we just determined in (9.8) can be shown to agree with the correlation kernel $K_{N}(x, y)$ obtained by the authors in [7, Theorem 3.2], for a particular choice of $b$. This agreement is not surprising, in view of the identification made after (2.17). Formula (9.8) for our kernel gives

$$
K_{n, m=2}(r=2, x ; s=2, y ; b)=\sum_{p=0}^{n-1} \frac{\Gamma\left(\nu_{1}+p+1\right)}{\Gamma^{2}\left(\nu_{1}+1\right) p !} P_{r=2, p}(x) Q_{s=2, p}(y) .
$$

Equation (9.12) implies that the function $P_{r=2, p}(x)$ can be written as

$$
P_{r=2, p}(x)=\sum_{j=0}^{p} \frac{(-p)_{j}}{\left(\nu_{1}+1\right)_{j}} \frac{x^{\frac{j}{2}} I_{j}\left(2 b x^{\frac{1}{2}}\right)}{b^{j} j !} .
$$

Now let us find a convenient representation for the function $Q_{s=2, p}(x)$. Equation (9.10) reads

$$
Q_{s=2, p}(x)=\sum_{j=0}^{p} \phi_{0, s=2}(j+1, y) \frac{(-p)_{j}}{\left(\nu_{1}+1\right)_{j} j !} .
$$

The function $\phi_{0, s=2}(j+1, y)$ was obtained in Proposition 9.1, see equation (9.3), and take into account that $s=m=2$. Using the representation of the exponential function in terms of the Meijer $G$-function

$$
x^{\nu} e^{-\frac{y}{x}}=G_{0,1}^{1,0}\left(\begin{array}{c|c}
- & x
\end{array}\right)
$$

we have

$$
\phi_{0, s=2}(j+1, y)=\int_{0}^{\infty} G_{0,1}^{1,0}\left(\begin{array}{c}
- \\
\nu_{1}+j
\end{array} \mid t\right) e^{-\frac{y}{t}-b^{2} t} \frac{d t}{t}=2\left(\frac{y}{b^{2}+1}\right)^{\frac{\nu_{1}+j}{2}} K_{\nu_{1}+j}\left(2 \sqrt{\left(b^{2}+1\right) y}\right) .
$$

Here, we have used (2.16). If we insert (9.29) in formula (9.27), we find

$$
Q_{s=2, p}(x)=2 \sum_{j=0}^{p} \frac{(-p)_{j}}{\left(\nu_{1}+1\right)_{j} j !}\left(\frac{y}{b^{2}+1}\right)^{\frac{\nu_{1}+j}{2}} K_{\nu_{1}+j}\left(2 \sqrt{\left(1+b^{2}\right) y}\right) .
$$

Thus the kernel $K_{n, 2}(2, x ; 2, y ; b)$ is given by equation (9.25), where the functions $P_{2, p}(x)$ and $Q_{2, p}(y)$ are defined by equations (9.26) and (9.30), respectively. 
Next, we turn to the kernel $K_{N}(x, y)$ obtained in [7, Theorem 3.2]. There, the parameters $\alpha(\mu)$ and $\delta(\mu)$ were defined as

$$
\alpha(\mu)=\frac{1+\mu}{2 \mu}, \quad \delta(\mu)=\frac{1-\mu}{2 \mu},
$$

where $\mu$ takes values in the interval $(0,1]$. We thus have

$$
\frac{\alpha(\mu)^{2}-\delta(\mu)^{2}}{\delta(\mu)}=\frac{2}{1-\mu}, \quad \frac{\alpha(\mu)^{2}-\delta(\mu)^{2}}{\alpha(\mu)}=\frac{2}{1+\mu}, \quad \alpha(\mu)^{2}-\delta(\mu)^{2}=\frac{1}{\mu} .
$$

Taking this into account, and identifying $\nu_{1}=\nu$ there, we see that the correlation kernel $K_{N}(x, y)$ in [7, Theorem 3.2] can be written as

$$
K_{N}(x, y)=\sum_{n=0}^{N-1} P_{n}(x) Q_{n}(y)
$$

where

$$
P_{n}(x)=\frac{(-1)^{n}\left(\nu_{1}+n\right) ! n !}{\sqrt{\mu}} \sum_{k=0}^{n} \frac{(-n)_{k}}{\left(\nu_{1}+k\right) ! k !}\left(\frac{2 x^{\frac{1}{2}}}{1-\mu}\right)^{k} I_{k}\left(\frac{1-\mu}{\mu} x^{\frac{1}{2}}\right)
$$

and

$$
Q_{n}(y)=\frac{2(-1)^{n}}{\sqrt{\mu}(n !)^{2}} \sum_{l=0}^{n} \frac{(-n)_{l}}{\left(\nu_{1}+l\right) ! l !}\left(\frac{2 y^{\frac{1}{2}}}{1+\mu}\right)^{l+\nu_{1}} K_{l+\nu_{1}}\left(\frac{1+\mu}{\mu} y^{\frac{1}{2}}\right)
$$

Setting

$$
b=\frac{1-\mu}{2 \sqrt{\mu}}, \quad x=\frac{\zeta}{\mu}, \quad y=\frac{\eta}{\mu},
$$

equations (9.26) and (9.30) take the form

$$
P_{r=2, p}(x)=\sum_{j=0}^{p} \frac{(-p)_{j}}{\left(\nu_{1}+1\right)_{j} j !}\left(\frac{2 \zeta^{\frac{1}{2}}}{1-\mu}\right)^{j} I_{j}\left(\frac{1-\mu}{\mu} \zeta^{\frac{1}{2}}\right),
$$

and

$$
Q_{s=2, p}(y)=2 \sum_{j=0}^{p} \frac{(-p)_{j}}{\left(\nu_{1}+1\right)_{j} j !}\left(\frac{2 \eta^{\frac{1}{2}}}{1+\mu}\right)^{j+\nu_{1}} K_{j+\nu_{1}}\left(\frac{1+\mu}{\mu} \eta^{\frac{1}{2}}\right) .
$$

Here, we used that $\left(\nu_{1}+1\right)_{j}=\left(\nu_{1}+j\right) ! / \nu_{1}$ !. Thus we obtain the following identity

$$
\mu K_{n, m=2}\left(r=2, \frac{\zeta}{\mu} ; s=2, \frac{\eta}{\mu} ; \frac{1-\mu}{2 \sqrt{\mu}}\right)=K_{N=n}(\zeta, \eta) .
$$

The extra factor of $\mu$ in front of the kernel on the left-hand side is due to the change of variables defined by equation (9.34). 


\section{Proof of Theorem 3.2}

Given the double contour integral representation for correlation kernel $K_{n, m}(r, x ; s, y ; b)$, we are ready to study the asymptotic of the Ginibre product process with coupling, i.e. the asymptotic of the matrix product process associated with the multi-matrix model (2.19). Recall that we consider the coupling parameter $b$ as a function of $n$, and investigate the hard edge scaling limit at the origin in different asymptotic regimes.

10.1. The weak coupling regime. In this regime we assume that $b(n) / \sqrt{n} \rightarrow 0$ as $n \rightarrow \infty$. Let us first establish equation (3.3). Consider the first term in the right-hand side of equation (2.37). We will show that in the weak coupling regime the hard edge scaling limit of the first term is given by

$$
\lim _{n \rightarrow \infty} \frac{1}{n} \phi_{r, s}\left(\frac{x}{n}, \frac{y}{n} ; b(n)\right)=\frac{1}{x} G_{0, s-r}^{s-r, 0}\left(\begin{array}{c|c}
- & \frac{y}{x}
\end{array}\right) \mathbf{1}_{s>r} .
$$

The explicit formula for $\phi_{r, s}(x, y ; b)$ is given in the statement of Theorem 2.8. Using this explicit formula, we immediately see that equation (10.1) holds true for $1 \leq r<s \leq$ $m-1$, and for $1 \leq s \leq r \leq m$. For $r=m-1, s=m$ the left hand side of equation (10.1) is equal to $\frac{1}{x} e^{-\frac{y}{x}}$, which can be rewritten as

$$
\frac{1}{x} G_{0,1}^{1,0}\left(\begin{array}{c|c}
- & y \\
\nu_{m} & x
\end{array}\right)=x^{\nu_{m}-1} e^{-\frac{y}{x}}
$$

(recall that $\nu_{m}=0$ ). Therefore, equation (10.1) holds true for $r=m-1, s=m$ as well. For $1 \leq r \leq m-2, s=m$ we can write

$$
\frac{1}{n} \phi_{r, s}\left(\frac{x}{n}, \frac{y}{n} ; b(n)\right)=\frac{1}{x} \int_{0}^{\infty} G_{0, m-r-1}^{m-r-1,0}\left(\begin{array}{c|c}
- & n t \\
\nu_{r+1}, \ldots, \nu_{m-1} & \frac{n t}{x}
\end{array}\right) e^{-\frac{y}{n t}-b^{2}(n) t} \frac{d t}{t} .
$$

Changing the integration variable as $t=\frac{\tau}{n}$, and taking into account that $e^{-\frac{b^{2}(n)}{n} \tau} \rightarrow 1$, in the weak coupling regime we obtain that equation (10.1) holds true for $1 \leq r \leq m-2$, $s=m$. In this calculations the procedure of taking the limit inside the integral can be justified by the dominated convergence theorem.

It remains to show that

$$
\begin{aligned}
& \lim _{n \rightarrow \infty}\left\{\frac{1}{n} S_{n, m}\left(r, \frac{x}{n} ; s, \frac{y}{n} ; b(n)\right)\right\} \\
& =\frac{1}{(2 \pi i)^{2}} \int_{-\frac{1}{2}-i \infty}^{-\frac{1}{2}+i \infty} d u \oint_{\Sigma_{\infty}} d t \frac{\prod_{j=0}^{s} \Gamma\left(u+\nu_{j}+1\right)}{\prod_{j=0}^{r} \Gamma\left(t+\nu_{j}+1\right)} \frac{\sin \pi u}{\sin \pi t} \frac{x^{t} y^{-u-1}}{u-t}
\end{aligned}
$$

where $\Sigma_{\infty}$ is a contour starting from $+\infty$ in the upper half plane and returning to $+\infty$ in the lower half plane, leaving $-\frac{1}{2}$ on the left, and encircling $\{0,1,2, \ldots\}$. To see that equation (10.3) indeed holds true observe that for $r, s \in\{1, \ldots, m-1\}$ the functions $p_{r}\left(t, \frac{x}{n} ; b(n)\right)$ and $q_{s}\left(u, \frac{y}{n} ; b(n)\right)$ are both identically equal to 1 , and that for 
$r, s \in\{1, \ldots, m-1\}$ equation (3.3) can be obtained by applying the same arguments as in Kuijlaars and Zhang [31, Section 5.2.]. In addition, we have

$$
p_{m}\left(t, \frac{x}{n} ; b(n)\right)=\frac{\Gamma(t+1) I_{t}\left(2 \frac{b(n)}{\sqrt{n}} x^{\frac{1}{2}}\right)}{\left(\frac{b(n)}{\sqrt{n}} x^{\frac{1}{2}}\right)^{t}} \simeq 1 ; \text { as } n \rightarrow \infty, \text { and } \frac{b(n)}{n^{\frac{1}{2}}} \rightarrow 0,
$$

and

$$
q_{m}\left(u+1, \frac{y}{n} ; b(n)\right)=\frac{2\left(\frac{b(n)}{\sqrt{n}} y^{\frac{1}{2}}\right)^{u} K_{u}\left(2 \frac{b(n)}{\sqrt{n}} y^{\frac{1}{2}}\right)}{\Gamma(u)} \simeq 1 ; \text { as } n \rightarrow \infty, \text { and } \frac{b(n)}{n^{\frac{1}{2}}} \rightarrow 0 .
$$

Therefore, equation (10.3) remains true for all $r, s \in\{1, \ldots, m\}$. In these calculations the procedure of taking the limit inside the double integral can be justified as in the proof of Theorem 5.3 in Kujlaars and Zhang [31] using the dominated convergence theorem, and the asymptotic properties of the involved Gamma and Bessel functions.

Now, the correlation kernel of the scaled Ginibre product process with coupling, formed by the configurations $\left(n y_{1}^{m}, \ldots, n y_{n}^{m} ; \ldots, n y_{1}^{1}, \ldots, n y_{n}^{1}\right)$, is related to the correlation kernel of the unscaled process with configurations $\left(y_{1}^{m}, \ldots, y_{n}^{m} ; \ldots, y_{1}^{1}, \ldots, y_{n}^{1}\right)$ by replacing $K_{n, m}(r, x ; s, y ; b(n))$ with $\frac{1}{n} K_{n, m}\left(r, \frac{x}{n} ; s, \frac{y}{n} ; b(n)\right)$. Taking this into account, we see that equation (3.3) implies the statement of Theorem 3.2 in the weak coupling regime.

10.2. The intermediate coupling regime. The proof of Theorem 3.2 for the intermediate coupling regime is based on arguments similar to those presented in Section 10.1. The difference is that in the intermediate coupling regime we have for the transition functions that depend on $b(N)$, that is for $r=m$ or $s=m$ :

$$
\lim _{n \rightarrow \infty}\left(\frac{1}{n} \phi_{r, s}\left(\frac{x}{n}, \frac{y}{n} ; b(n)\right)\right)=\phi_{r, s}(x, y ; \alpha) .
$$

This can be seen immediately from the formulae for $\phi_{r, s}(x, y ; b(n))$ in the statement of Theorem 2.8 when $n \rightarrow \infty$ and $\frac{b(n)}{n^{\frac{1}{2}}} \rightarrow \alpha$, as well as

$$
\begin{aligned}
& p_{m}\left(t, \frac{x}{n} ; b(n)\right) \rightarrow p_{m}(t, x ; \alpha), \\
& q_{m}\left(u, \frac{y}{n} ; b(n)\right) \rightarrow q_{m}(u, y ; \alpha) .
\end{aligned}
$$

using (2.40) and (2.41).

10.3. The strong coupling regime. Let us first establish the limiting relations for the correlation kernel in the strong coupling regime, equations (3.6)-(3.8). The kernels with levels $1 \leq r, s \leq m-1$ are $b$-independent, and the limit was already established in (3.3).

In order to establish equations (3.6)-(3.8) we need to take into account that $p_{m}(t, x ; b)$ and $q_{m}(u, y ; b)$ are nontrivial expressions involving the modified Bessel functions of the 
first and of the second kind, see equations (2.40), (2.41). These functions have the following asymptotic as $b(n) / n^{\frac{1}{2}} \rightarrow \infty$ :

$$
p_{m}\left(t, \frac{b^{2}(n) x^{2}}{n^{2}} ; b(n)\right) \sim \frac{\Gamma(t+1)}{2 \pi^{\frac{1}{2}}} \frac{e^{2 \frac{b^{2}(n)}{n} x}}{\left(\frac{b^{2}(n)}{n} x\right)^{t+\frac{1}{2}}},
$$

and

$$
q_{m}\left(u+1, \frac{b^{2}(n) y^{2}}{n^{2}} ; b(n)\right) \sim \frac{\pi^{\frac{1}{2}}}{\Gamma(u+1)} \frac{b^{2 u+1}(n)}{n^{u+\frac{1}{2}}} y^{u+\frac{1}{2}} e^{-2 \frac{b^{2}(n)}{n} y}
$$

The asymptotic formulae just written above follow immediately from the known asymptotic of the modified Bessel function of the first kind $I_{t}\left(2 b x^{\frac{1}{2}}\right)$, and from the known asymptotic of the modified Bessel function of the second kind $K_{u}\left(2 b y^{\frac{1}{2}}\right)$ as $b \rightarrow \infty$, see equations (2.13) and (2.17), respectively. In addition, we have the following ratio asymptotic of Gamma functions

$$
\frac{\Gamma(t-n+1)}{\Gamma(u-n+1)}=\frac{\sin \pi u}{\sin \pi t} n^{t-u}\left(1+O\left(n^{-1}\right)\right), \text { as } n \rightarrow \infty .
$$

Using the asymptotic formulae mentioned above we obtain the following limiting relations.

- For $1 \leq r \leq m-1, s=m$ we have

$$
\begin{aligned}
& \lim _{n \rightarrow \infty} \frac{2^{\frac{1}{2}} b(n) y^{\frac{1}{2}}}{n^{\frac{3}{2}}} S_{n, m}\left(r, \frac{x}{n} ; m, \frac{b^{2}(n) y^{2}}{n^{2}} ; b(n)\right) \frac{e^{2 \frac{b^{2}(n)}{n} y}}{2^{\frac{1}{2}} \pi^{\frac{1}{2}}} \\
& =\frac{1}{(2 \pi i)^{2}} \int_{-\frac{1}{2}-i \infty}^{-\frac{1}{2}+i \infty} d u \oint_{\Sigma_{\infty}} d t \frac{\prod_{j=0}^{m-1} \Gamma\left(u+\nu_{j}+1\right)}{\prod_{j=0}^{r} \Gamma\left(t+\nu_{j}+1\right)} \frac{\sin \pi u}{\sin \pi t} \frac{x^{t} y^{-u-1}}{u-t} .
\end{aligned}
$$

- For $r=m, 1 \leq s \leq m-1$ we have

$$
\begin{aligned}
& \lim _{n \rightarrow \infty} \frac{2^{\frac{1}{2}} b(n) x^{\frac{1}{2}}}{n^{\frac{3}{2}}} S_{n, m}\left(m, \frac{b^{2}(n) x^{2}}{n^{2}} ; s, \frac{y}{n} ; b(n)\right) \frac{2^{\frac{1}{2}} \pi^{\frac{1}{2}}}{e^{2 \frac{b^{2}(n)}{n} x}} \\
& =\frac{1}{(2 \pi i)^{2}} \int_{-\frac{1}{2}-i \infty}^{-\frac{1}{2}+i \infty} d u \oint_{\Sigma_{\infty}} d t \frac{\prod_{j=0}^{s} \Gamma\left(u+\nu_{j}+1\right)}{\prod_{j=0}^{m-1} \Gamma\left(t+\nu_{j}+1\right)} \frac{\sin \pi u}{\sin \pi t} \frac{x^{t} y^{-u-1}}{u-t} .
\end{aligned}
$$


- Finally, for $r=m$ and $s=m$ the following limiting relation holds true

$$
\begin{aligned}
& \lim _{n \rightarrow \infty} \frac{2 b^{2}(n) x^{\frac{1}{2}} y^{\frac{1}{2}}}{n^{2}} S_{n, m}\left(m, \frac{b^{2}(n) x^{2}}{n^{2}} ; m, \frac{b^{2}(n) y^{2}}{n^{2}} ; b(n)\right) \frac{e^{2 \frac{b^{2}(n)}{n} y}}{e^{2 \frac{b^{2}(n)}{n} x}} \\
& =\frac{1}{(2 \pi i)^{2}} \int_{-\frac{1}{2}-i \infty}^{-\frac{1}{2}+i \infty} d u \oint_{\Sigma_{\infty}} d t \frac{\prod_{j=0}^{m-1} \Gamma\left(u+\nu_{j}+1\right)}{\prod_{j=0}^{m-1} \Gamma\left(t+\nu_{j}+1\right)} \frac{\sin \pi u}{\sin \pi t} \frac{x^{t} y^{-u-1}}{u-t} .
\end{aligned}
$$

In the derivation of these formulae the interchange of limits and integrals can be justified by the dominated convergence theorem in the same way as it is done in Kuijlaars and Zhang [31, Section 5.2.]. Formulae (10.8)-(10.10) give the scaling limits of the second term, $S_{n, m}(r, x ; s, y ; b(n))$, in equation (2.37) for the correlation kernel $K_{n, m}(r, x ; s, y ; b(n))$.

Now, let us find the asymptotic of the first term, $\phi_{r, s}(x, y ; b)$, in equation (2.37). Recall that we already know the asymptotic of $\phi_{r, s}(x, y ; b)$ for $1 \leq r, s \leq m-1$. So it is enough to consider two cases.

- The first case corresponds to $1 \leq r \leq m-2, s=m$. In this case we have

$$
\begin{aligned}
& \frac{2^{\frac{1}{2}} b(n) y^{\frac{1}{2}}}{n^{\frac{3}{2}}} \phi_{r, m}\left(\frac{x}{n} ; \frac{b^{2}(n) y^{2}}{n^{2}} ; b(n)\right) \frac{e^{2^{\frac{b^{2}(n)}{n} y}}}{2^{\frac{1}{2}} \pi^{\frac{1}{2}}} \\
& =\frac{2^{\frac{1}{2} b(n) y^{\frac{1}{2}}}}{n^{\frac{3}{2}}} \frac{n}{x}\left[\int_{0}^{\infty} G_{0, m-r-1}^{m-r-1,0}\left(\begin{array}{c}
- \\
\nu_{r+1}, \ldots, \nu_{m-1}
\end{array} \mid \frac{n t}{x}\right) e^{-\frac{b^{2}(n) y^{2}}{n^{2} t}-b^{2}(n) t} \frac{d t}{t}\right] \frac{e^{2^{\frac{b^{2}(n)}{n}} y}}{2^{\frac{1}{2}} \pi^{\frac{1}{2}}} .
\end{aligned}
$$

The expression in the brackets can be written as

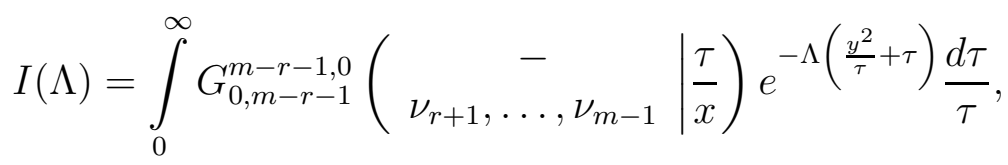

where $\Lambda=\frac{b^{2}(n)}{n}$. Using Laplace's method for asymptotic expansions of integrals (see, for example, Miller [36, Chapter 3.]) we obtain that the expression in the brackets has the following asymptotic

$$
\begin{aligned}
\int_{0}^{\infty} G_{0, m-r-1}^{m-r-1,0} & \left(\begin{array}{c}
- \\
\nu_{r+1}, \ldots, \nu_{m-1}
\end{array} \mid \frac{n t}{x}\right) e^{-\frac{b^{2}(n) y^{2}}{n^{2} t}-b^{2}(n) t} \frac{d t}{t} \\
& \sim \frac{\pi^{\frac{1}{2}} n^{\frac{1}{2}}}{b(n) y^{\frac{1}{2}}} G_{0, m-r-1}^{m-r-1,0}\left(\begin{array}{c}
- \\
\nu_{r+1}, \ldots, \nu_{m-1}
\end{array} \mid \frac{y}{x}\right) e^{-\frac{2 b^{2}(n) y}{n}}, \text { as } \frac{b(n)}{\sqrt{n}} \rightarrow \infty .
\end{aligned}
$$

This gives

$$
\lim _{n \rightarrow \infty} \frac{2^{\frac{1}{2}} b(n) y^{\frac{1}{2}}}{n^{\frac{3}{2}}} \phi_{r, m}\left(\frac{x}{n} ; \frac{b^{2}(n) y^{2}}{n^{2}} ; b(n)\right) \frac{e^{2 \frac{b^{2}(n)}{n} y}}{2^{\frac{1}{2}} \pi^{\frac{1}{2}}}=\frac{1}{x} G_{0, m-r-1}^{m-r-1,0}\left(\begin{array}{c|c}
- \\
\nu_{r+1}, \ldots, \nu_{m-1}
\end{array} \mid \frac{y}{x}\right),
$$


where $1 \leq r \leq m-2$.

- For $r=m-1, s=m$ we can write

$$
\begin{aligned}
\frac{2^{\frac{1}{2}} b(n) y^{\frac{1}{2}}}{n^{\frac{3}{2}}} \phi_{m-1, m}\left(\frac{x}{n} ; \frac{b^{2}(n) y^{2}}{n^{2}} ; b(n)\right) \frac{e^{2 \frac{b^{2}(n)}{n} y}}{2^{\frac{1}{2}} \pi^{\frac{1}{2}}} & =\frac{b(n) y^{\frac{1}{2}}}{\pi^{\frac{1}{2}} n^{\frac{3}{2}}} \frac{n}{x} e^{-2 \frac{b^{2}(n) y^{2}}{n x}-\frac{b^{2}(n) x}{n}+\frac{2 b^{2}(n) y}{n}} \\
& =\frac{b(n) y^{\frac{1}{2}}}{\pi^{\frac{1}{2}} n^{\frac{1}{2}} x} e^{-\frac{b^{2}(n)}{x n}(y-x)^{2}} .
\end{aligned}
$$

We conclude that

$$
\lim _{n \rightarrow \infty} \frac{2^{\frac{1}{2}} b(n) y^{\frac{1}{2}}}{n^{\frac{3}{2}}} \phi_{m-1, m}\left(\frac{x}{n} ; \frac{b^{2}(n) y^{2}}{n^{2}} ; b(n)\right) \frac{e^{2 \frac{b^{2}(n)}{n} y}}{2^{\frac{1}{2}} \pi^{\frac{1}{2}}}=\delta(x-y),
$$

representing the Dirac delta distribution. Taking into account equations (10.8)-(10.10), (10.13), and (10.15) we obtain the limiting relations (3.6)-(3.8) in the statement of Theorem 3.2 ,

In the weak and intermediate coupling regime in the previous two subsections the points on all levels were rescaled in the same way with $n$. In the strong coupling regime this is no longer so. Let us therefore explain how the different rescaling of the point configurations translates into different rescalings of the various correlation kernels. This justifies the different scalings that we have applied above. Assume that the point configurations

$$
\left(y_{1}^{m}, \ldots, y_{n}^{m} ; \ldots ; y_{1}^{1}, \ldots, y_{n}^{1}\right)
$$

form a determinantal process on $\{1, \ldots, m\} \times \mathbb{R}_{>0}$ defined by the correlation kernel $K_{n, m}(r, x ; s, y ; b)$. Consider the scaled determinantal process on $\{1, \ldots, m\} \times \mathbb{R}_{>0}$ formed by the scaled point configurations

$$
\begin{aligned}
& \left(\frac{n}{b(n)}\left(y_{1}^{m}\right)^{\frac{1}{2}}, \ldots, \frac{n}{b(n)}\left(y_{n}^{m}\right)^{\frac{1}{2}} ; n y_{1}^{m-1}, \ldots, n y_{n}^{m-1} ; \ldots ; n y_{1}^{m-1}, \ldots, n y_{n}^{m-1}\right) \\
& =\left(u_{1}^{m}, \ldots, u_{n}^{m} ; u_{1}^{m-1}, \ldots, u_{n}^{m-1} ; \ldots ; u_{1}^{1}, \ldots, u_{n}^{1}\right) .
\end{aligned}
$$

If $\varrho_{k_{1}, \ldots, k_{m}}$ is the correlation function of the original determinantal process, see their definition in (8.2), and $\widehat{\varrho}_{k_{1}, \ldots, k_{m}}$ is that of the scaled determinantal process, then we must have

$$
\begin{aligned}
& \varrho_{k_{1}, \ldots, k_{m}}\left(y_{1}^{1}, \ldots, y_{k_{1}}^{1} ; \ldots ; y_{1}^{m}, \ldots, y_{k_{m}}^{m}\right)\left(d y_{1}^{1}, \ldots, d y_{k_{1}}^{1}\right) \ldots\left(d y_{1}^{m}, \ldots, d y_{k_{m}}^{m}\right) \\
& =\widehat{\varrho}_{k_{1}, \ldots, k_{m}}\left(u_{1}^{1}, \ldots, u_{k_{1}}^{1} ; \ldots ; u_{1}^{m}, \ldots, u_{k_{m}}^{m}\right)\left(d u_{1}^{1}, \ldots, d u_{k_{1}}^{1}\right) \ldots\left(d u_{1}^{m}, \ldots, d u_{k_{m}}^{m}\right) .
\end{aligned}
$$


This gives the following relation between the initial correlation function, $\varrho_{k_{1}, \ldots, k_{m}}$, and the correlation function $\widehat{\varrho}_{k_{1}, \ldots, k_{m}}$ of the scaled determinant process

$$
\begin{aligned}
& \widehat{\varrho}_{k_{1}, \ldots, k_{m}}\left(u_{1}^{1}, \ldots, u_{k_{1}}^{1} ; \ldots ; u_{1}^{m-1}, \ldots, u_{k_{m-1}}^{m-1} ; u_{1}^{m}, \ldots, u_{k_{m}}^{m}\right) \\
& =\frac{2^{k_{m}} u_{1}^{m} \ldots u_{k_{m}}^{m}}{n^{k_{1}+\ldots+k_{m-1}}}\left(\frac{b(n)}{n}\right)^{2 k_{m}} \\
& \times \varrho_{k_{1}, \ldots, k_{m}}\left(\frac{u_{1}^{1}}{n}, \ldots, \frac{u_{k_{1}}^{1}}{n} ; \ldots ; \frac{u_{1}^{m-1}}{n}, \ldots, \frac{u_{k_{m-1}-1}^{m}}{n} ; \frac{b^{2}(n)}{n^{2}}\left(u_{1}^{m}\right)^{2}, \ldots, \frac{b^{2}(n)}{n^{2}}\left(u_{k_{m}}^{m}\right)^{2}\right) .
\end{aligned}
$$

If $K_{n, m}(r, u ; s, v)$ is the correlation kernel of the initial determinantal process, and $\widehat{K}_{n, m}(r, u ; s, v)$ is the correlation kernel of the scaled determinantal process, then relation (10.17) between the correlation functions implies

$$
\begin{aligned}
& \widehat{K}_{n, m}(r, u ; s, v) \\
& = \begin{cases}\frac{1}{n} K_{n, m}\left(r, \frac{u}{n} ; s, \frac{v}{n}\right), & 1 \leq r, s \leq m-1, \\
\frac{2^{\frac{1}{2}} b(n) v^{\frac{1}{2}}}{n^{\frac{3}{2}}} K_{n, m}\left(r, \frac{u}{n} ; m, \frac{b^{2}(n)}{n^{2}} v^{2}\right), & 1 \leq r \leq m-1, s=m, \\
\frac{2^{\frac{1}{2}} b(n) u^{\frac{1}{2}}}{n^{\frac{3}{2}}} K_{n, m}\left(m, \frac{b^{2}(n)}{n^{2}} u^{2} ; s, \frac{v}{n}\right), & r=m, 1 \leq s \leq m-1, \\
\frac{2 b^{2}(n) u^{\frac{1}{2}} v^{\frac{1}{2}}}{n^{2}} K_{n, m}\left(m, \frac{b^{2}(n)}{n^{2}} u^{2} ; m, \frac{b^{2}(n)}{n^{2}} v^{2}\right), & r=m, s=m .\end{cases}
\end{aligned}
$$

Also, let us take into account that if we have two correlation kernels, say $K_{n, m}(r, x ; s, y)$ and $K_{n, m}^{\prime}(r, x ; s, y)$ living on $\{1, \ldots, m\} \times \mathbb{R}_{>0}$, and related as

$$
K_{n, m}^{\prime}(r, x ; s, y)=\frac{\varphi_{r}(x)}{\varphi_{s}(y)} K_{n, m}(r, x ; s, y)
$$

where $\varphi_{1}, \ldots, \varphi_{m}$ are certain non-vanishing functions defined on $\mathbb{R}_{>0}$, then both $K_{n, m}(r, x ; s, y)$ and $K_{n, m}^{\prime}(r, x ; s, y)$ give the same correlation functions, and therefore define the same determinantal point process on $\{1, \ldots, m\} \times \mathbb{R}_{>0}$. The two kernels are therefore called equivalent kernels. In other words, we have from (10.16)

$$
\begin{aligned}
& \operatorname{det}\left[\begin{array}{ccc}
\left(K_{n, m}\left(1, x_{i}^{1} ; 1, x_{j}^{1}\right)\right)_{i=1, \ldots, k_{1}}^{j=1, \ldots, k_{1}} & \ldots & \left(K_{n, m}\left(1, x_{i}^{1} ; m, x_{j}^{m}\right)\right)_{i=1, \ldots, k_{1}}^{j=1, \ldots, k_{m}} \\
\vdots & & \\
\left(K_{n, m}\left(m, x_{i}^{m} ; 1, x_{j}^{1}\right)\right)_{i=1, \ldots, k_{m}}^{j=1, \ldots, k_{1}} & \ldots & \left(K_{n, m}\left(m, x_{i}^{m} ; m, x_{j}^{m}\right)\right)_{i=1, \ldots, k_{m}}^{j=1, \ldots, k_{m}}
\end{array}\right] \\
& =\operatorname{det}\left[\begin{array}{ccc}
\left(K_{n, m}^{\prime}\left(1, x_{i}^{1} ; 1, x_{j}^{1}\right)\right)_{i=1, \ldots, k_{1}}^{j=1, \ldots, k_{1}} & \ldots & \left(K_{n, m}^{\prime}\left(1, x_{i}^{1} ; m, x_{j}^{m}\right)\right)_{i=1, \ldots, k_{1}}^{j=1, \ldots, k_{m}} \\
\vdots & & \\
\left(K_{n, m}^{\prime}\left(m, x_{i}^{m} ; 1, x_{j}^{1}\right)\right)_{i=1, \ldots, k_{m}}^{j=1, \ldots, k_{1}} & \ldots & \left(K_{n, m}^{\prime}\left(m, x_{i}^{m} ; m, x_{j}^{m}\right)\right)_{i=1, \ldots, k_{m}}^{j=1, \ldots, k_{m}}
\end{array}\right] .
\end{aligned}
$$


Therefore, the correlation kernel $\widehat{K}_{n, m}^{\prime}(r, x ; s, y)$ defined by

$$
\begin{aligned}
& \widehat{K}_{n, m}^{\prime}(r, u ; s, v) \\
& = \begin{cases}\frac{1}{n} K_{n, m}\left(r, \frac{u}{n} ; s, \frac{v}{n}\right), & 1 \leq r, s \leq m-1, \\
\frac{2^{\frac{1}{2}} b(n) v^{\frac{1}{2}}}{n^{\frac{3}{2}}} K_{n, m}\left(r, \frac{u}{n} ; m, \frac{b^{2}(n)}{n^{2}} v^{2}\right) \frac{e^{2 \frac{b^{2}(n)}{n} v}}{2^{\frac{1}{2}} \pi^{\frac{1}{2}}}, & 1 \leq r \leq m-1, s=m, \\
\frac{2^{\frac{1}{2}} b(n) u^{\frac{1}{2}}}{n^{\frac{3}{2}}} K_{n, m}\left(m, \frac{b^{2}(n)}{n^{2}} u^{2} ; s, \frac{v}{n}\right) \frac{2^{\frac{1}{2}} \pi^{\frac{1}{2}}}{e^{\frac{b^{2}(n)}{n}} u}, & r=m, 1 \leq s \leq m-1, \\
\frac{2 b^{2}(n) u^{\frac{1}{2}} v^{\frac{1}{2}}}{n^{2}} K_{n, m}\left(m, \frac{b^{2}(n)}{n^{2}} u^{2} ; m, \frac{b^{2}(n)}{n^{2}} v^{2}\right) \frac{e^{2 \frac{b^{2}(n)}{n} v}}{e^{\frac{b^{2}(n)}{n}},}, & r=m, s=m,\end{cases}
\end{aligned}
$$

gives the same scaled determinantal process as the correlation kernel $\widehat{K}_{n, m}(r, x ; s, y)$ defined by equation (10.18). We conclude that the limiting relations (3.6)-(3.8) imply that the scaled determinantal process on $\{1, \ldots, m\} \times \mathbb{R}_{>0}$ formed by the scaled point configurations

$$
\left(\frac{n}{b(n)}\left(y_{1}^{m}\right)^{\frac{1}{2}}, \ldots, \frac{n}{b(n)}\left(y_{n}^{m}\right)^{\frac{1}{2}} ; n y_{1}^{m-1}, \ldots, n y_{n}^{m-1} ; \ldots ; n y_{1}^{m-1}, \ldots, n y_{n}^{m-1}\right)
$$

converges as $n \rightarrow \infty$ to the determinantal point process $\mathcal{P}_{\infty, m-1}^{\text {Ginibre }}$ on $\{1, \ldots, m-1\} \times \mathbb{R}_{>0}$ with the identification of levels $m$ and $m-1$ as given in Theorem 3.2 (C).

\section{Proof of Theorem 4.1}

Consider the determinantal point process on $\{1, \ldots, m\} \times \mathbb{R}_{>0}$ defined by the correlation kernel $K_{\infty, m}^{\text {interpol }}(r, x ; s, y ; \alpha)$, see equation (3.4). From equation (2.38) we obtain that

$$
\lim _{\alpha \rightarrow 0} \phi_{r, s}(x, y ; \alpha)=\frac{1}{x} G_{0, s-r}^{s-r, 0}\left(\begin{array}{c|c}
- & \frac{y}{x}
\end{array}\right) \mathbf{1}_{s>r} .
$$

Here, we have used the fact that $\nu_{m}=0$, the integration formula

$$
\int_{0}^{\infty} G_{0, m-r-1}^{m-r-1,0}\left(\begin{array}{c|c}
- & \frac{y}{t}
\end{array}\right) t^{\nu_{m}} e^{-t} \frac{d t}{t}=G_{0, m-r}^{m-r, 0}\left(\begin{array}{c}
- \\
\nu_{r+1}, \ldots, \nu_{m-1}, \ldots, \nu_{m}
\end{array} \mid y\right),
$$

together with the representation of the exponential function in terms of the Meijer $G$ function (9.28). Thus, as $\alpha \rightarrow 0$, the first term in the right-hand side of equation (3.4) for the correlation kernel $K_{\infty, m}^{\text {interpol }}(r, x ; s, y ; \alpha)$ turns into the first term in the right-hand side of equation (3.1) for the correlation kernel $K_{\infty, m}^{\text {Ginibre }}(r, x ; s, y)$. Moreover, taking into account that

$$
\lim _{\alpha \rightarrow 0} p_{m}(t, x ; \alpha)=1, \quad \lim _{\alpha \rightarrow 0} q_{m}(u, y ; \alpha)=1,
$$

from equations (2.7) and (2.24), we see that the second term in formula (3.4) for $K_{\infty, m}^{\text {interpol }}(r, x ; s, y ; \alpha)$ turns into the second term in formula (3.1) for $K_{\infty, m}^{\text {Ginibre }}(r, x ; s, y)$. In these calculations the procedure of taking the limit inside the double integral can be justified using the dominated convergence theorem. Thus Theorem 4.1 (A) is proved. 
The proof of Theorem 4.1(B) is very similar to that of Theorem 3.2 (C). For this reason we present the sketch of the proof of Theorem4.1(B) only. For $1 \leq r, s \leq m-1$ the kernel does not depend on $\alpha$ and agrees with that of the Ginibre point process given by equation (3.1). In order to check equations (4.1)-(4.3) we use the known asymptotic formulae for the modified Bessel functions of the first and of the second kind (see equations (2.13) and (2.17)) to deduce that

$$
p_{m}\left(t, \alpha^{2} x^{2} ; \alpha\right) \sim \frac{\Gamma(t+1)}{\left(\alpha^{2} x\right)^{t}} \frac{e^{2 \alpha^{2} x}}{2 \pi^{\frac{1}{2}} \alpha x^{\frac{1}{2}}}, \quad \alpha \rightarrow \infty,
$$

and that

$$
q_{m}\left(u+1, \alpha^{2} y^{2} ; \alpha\right) \sim \frac{\pi^{\frac{1}{2}}}{\Gamma(u+1)} \frac{\left(\alpha^{2} y\right)^{u+1}}{\alpha y^{\frac{1}{2}}} e^{-2 \alpha^{2} y}, \quad \alpha \rightarrow \infty .
$$

Similar arguments as in the proof of Theorem 3.2 (C), can be applied to show that equations (4.1)-(4.3) ensure the convergence of the scaled interpolating process to $\mathcal{P}_{\infty, m-1}^{\text {Ginibre }}$.

\section{REFERENCES}

[1] Adhikari, K.; Kishore Reddy, N.; Ram Reddy, T.; Saha, K. Determinantal point processes in the plane from products of random matrices. Ann. Inst. Henri Poincaré Probab. Stat. 52 (2016), no. $1,16-46$.

[2] Akemann, G.; Burda, Z. Universal microscopic correlation functions for products of independent Ginibre matrices. J. Phys. A: Math. Theor. 45 (2012) 465201.

[3] Akemann, G.; Checinski, T.; Liu, D.-Z.; Strahov, E. Finite rank perturbations in products of coupled random matrices: From one correlated to two Wishart ensembles. arXiv:1704.05224

[4] Akemann, G.; Kieburg M.; Wei, L. Singular value correlation functions for products of Wishart random matrices. J. Phys. A. 46 (2013) 275205, 24pp.

[5] Akemann, G.; Ipsen, J.; Kieburg M. Products of rectangular random matrices: singular values and progressive scattering. Phys. Rev. E 88 (2013) 052118, 14pp.

[6] Akemann, G.; Ipsen, J.; Strahov, E. Permanental processes from products of complex and quaternionic induced Ginibre ensembles. Random Matrices Theory Appl. 3 (2014), no. 4, 1450014, 54.

[7] Akemann, G.; Strahov, E. Dropping the independence: singular values for products of two coupled random matrices. Commun. Math. Phys. 345 (2016), no. 1, 101-140.

[8] Akemann, G.; Strahov, E. Hard edge limit of the product of two strongly coupled random matrices. Nonlinearity 29 (2016) 3743-3776

[9] Ambjørn, J,; Chekov, L. The matrix model for hypergeometric Hurwitz numbers. Theor.Math. Phys. 181, Issue 3 (2014), 1486-1498.

[10] Bertola, M.; Bothner, T. Universality conjecture and results for a model of several coupled positivedefinite matrices. Commun. Math. Phys. 337 (2015), no. 3, 1077-1141.

[11] Bertola, M.; Eynard, B.; Harnad, J. Duality, Biorthogonal Polynomials and Multi-Matrix Models Commun. Math. Phys. 229 (2002), no. 1, 73-120.

[12] Bertola, M.; Gekhtman, M.; Szmigielski, J. The Cauchy two-matrix model. Commun. Math. Phys. 287 (2009), no. 3, 983-1014.

[13] Bertola, M.; Gekhtman, M.; Szmigielski, J. Strong asymptotics for Cauchy biorthogonal polynomials with application to the Cauchy two-matrix model. J. Math. Phys. 54 (2013), no. 4, 043517, 25

[14] Bertola, M.; Gekhtman, M.; Szmigielski, J. Cauchy-Laguerre two-matrix model and the Meijer-G random point field. Commun. Math. Phys. 326 (2014), no. 1, 111-144. 
[15] Borodin, A. Deteminantal point processes. Chapter 11, The Oxford Handbook of Random Matrix Theory (Eds. G. Akemann, J. Baik, P. Di Francesco). Oxford Unversity Press, Oxford, (2011), 231-249.

[16] Brower, R.; Rossi, P; Tan, C.-I., The external field problem for QCD. Nuclear Phys. B 190 [FS3] (1981) no. 4, 699-718.

[17] Eynard, B.; Kimura, T.; Ribault, S. Random matrices. arXiv:1510.04430.

[18] Eynard, B.; Mehta, M. L. Matrices coupled in a chain. I. Eigenvalue correlations. J. Phys. A 31 (1998), no. 19, 4449-4456.

[19] Filev, V. G.; O'Connor, D. Multi-matrix models at general coupling. J. Phys. A 46 (2013), no. 47, 475403, $21 \mathrm{pp}$.

[20] Fischmann, J.; Bruzda, W.; Khoruzhenko, B.A.; Sommers, H.-J.; Zyczkowski, K. Induced Ginibre ensemble of random matrices and quantum operations. J. Phys. A 45 (2012), no. 7, 075203.

[21] Forrester, P. J. Eigenvalue statistics for product complex Wishart matrices. J. Phys. A 47 (2014), no. 34,345202 .

[22] Forrester, P.J.; Ipsen, J.R. Real eigenvalue statistics for products of asymmetric real Gaussian matrices. Lin. Alg. Appl. 510 (2016), 259-290

[23] Gradshteyn, I.S.; Ryzhik, I.M. Table of Integrals, Series, and Products. A. Jeffrey and D. Zwillinger (eds.). Sixth edition (2000).

[24] Harish-Chandra. Differential operators on a semisimple Lie algebra, Am. J. Math. 79 (1957) 87-120.

[25] Ipsen, J. Products of independent quaternion Ginibre matrices and their correlation functions. J. Phys. A 46 (2013), no. 26, 265201.

[26] Ismail, M. E. H. Classical and quantum orthogonal polynomials in one variable. Encyclopedia of Mathematics and its Applications, 98. Cambridge University Press, Cambridge, 2009.

[27] Itzykson C. and Zuber, J.B. The Planar Approximation 2. J. Math. Phys. 21 (1980) 411.

[28] Johansson, K. Random matrices and determinantal processes. Lecture notes of the Les Houches Summer School Session LXXXIII, 2005. Mathematical Statistical Physics (Elsevier B. V, Amsterdam, 2006) 1-55.

[29] Johansson, K. From Gumbel to Tracy-Widom. Probab. Theory Related Fields 138, no. 1-2 (2007) $75-112$.

[30] Kuijlaars A.B.J.; Stivigny, D. Singular values of products of random matrices and polynomial ensembles. Random Matrices: Theory and Applications 3 (2014) 1450011.

[31] Kuijlaars, A.B.J.; Zhang, L. Singular values of products of Ginibre random matrices, multiple orthogonal polynomials and hard edge scaling limits. Commun. Math. Phys. 332 (2014) 759-781.

[32] Leutwyler, H.; Smilga, A. Spectrum of Dirac operator and role of winding number in QCD. Phys. Rev. D 46 (1992) 5607.

[33] Liu, D.-Z. Singular values for products of two coupled random matrices: hard edge phase transition. Constr. Approx. (2017). https://doi.org/10.1007/s00365-017-9389-z.

[34] Liu, D-Z.; Wang, D.; Zhang, L. Bulk and soft-edge universality for singular values of products of Ginibre random matrices. Ann. Inst. Henri Poincaré, Prob. Stat. 52, No. 4 (2016) 1734-1762.

[35] Luke, Y.L. The special functions and their approximations. Academic Press, New York 1969.

[36] Miller, P. D. Applied Asymptotic Analysis. Graduate Studies in Mathematics. Volume 75. American Mathematical Society.

[37] Moshe, M.; Neuberger, H.; Shapiro, B. Generalized ensemble of random matrices. Phys. Rev. Lett. 73 (1994) 1497-1500.

[38] Olver, F.W.L et al. (Eds.), NIST Handbook of Mathematical Functions. Cambridge University Press, Cambridge 2010.

[39] Osborn, J.C. Universal results from an alternate random matrix model for QCD with a baryon chemical potential. Phys. Rev. Lett. 93 (2004) 222001.

[40] Pandey, A.; Mehta, M. L. Gaussian ensembles of random Hermitian matrices intermediate between orthogonal and unitary ones. Commun. Math. Phys. 87, no. 4 (1982/83), 449-468. 
[41] Strahov, E. Dynamical correlation functions for products of random matrices. Random Matrices Theory Appl. 4 (2015), no. 4, 1550020.

[42] Tracy, C. A.; Widom, H. Differential equations for Dyson processes. Commun. Math. Phys. 252 (2004), 7-41.

Faculty of Physics, Bielefeld University, P.O. Box 100131, 33501 Bielefeld, Germany

E-mail address: akemann@physik.uni-bielefeld.de

Department of Mathematics, The Hebrew University of Jerusalem, Givat Ram, Jerusalem 91904, ISRAEL

E-mail address: strahov@math.huji.ac.il 